ThE ASTROPHYSiCAL JOURNAL, 450:217-232, 1995 September 1 0 1995. The American Astronomical Society. Alt rights reserved. Printed in U.S.A.

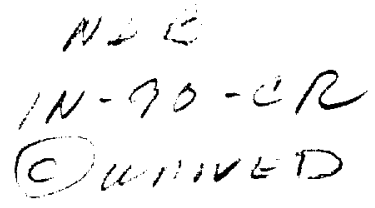

\title{
SPECTRAL AND TEMPORAL CHARACTERISTICS OF X-RAY-BRIGHT STARS IN THE PLEIADES
}

\author{
Marc Gagne, ${ }^{1,2}$ Jean-Pierre Caillault, ${ }^{1}$ and John R. Stauffer ${ }^{3}$ \\ Received 1995 March 8; accepted 1995 March 13
}

\begin{abstract}
We follow up our deep ROSAT imaging survey of the Pleiades (Stauffer et al. 1994) with an analysis of the spectral and temporal characteristics of the X-ray-bright stars in the Pleiades. Raymond \& Smith (1977) oneand two-temperature models have been used to fit the position-sensitive proportional counter (PSPC) pulseheight spectra of the dozen or so brightest sources associated with late-type Pleiades members. The best-fit temperatures suggest hot coronal temperatures for $\mathbf{K}, \mathbf{M}$, and rapidly rotating $\mathbf{G}$ stars, and cooler temperatures for $F$ and slowly rotating $G$ stars. In order to probe the many less $X$-ray-luminous stars, we have generated composite spectra by combining net counts from all Pleiades members according to spectral type and rotational velocity. Model fits to the composite spectra confirm the trend seen in the individual spectral fits. Particularly interesting is the apparent dependence of coronal temperature on $L_{\mathrm{x}} / L_{\mathrm{bol}}$. A hardness-ratio analysis also confirms some of these trends. The PSPC data have also revealed a dozen or so strong $X$-ray flares with peak X-ray luminosities in excess of $\sim 10^{30}$ ergs $s^{-1}$. We have modeled the brightest of these flares with a simple quasi-static cooling loop model. The peak temperature and emission measure and the inferred electron density and plasma volume suggest a very large scale flaring event. The PSPC data were collected over a period of $\sim 18$ months, allowing us to search for source variability on timescales ranging from less than a day (in the case of flares) to more than a year between individual exposures. On approximately year-long timescales, roughly $25 \%$ of the late-type stars are variable. Since the Pleiades was also intensively monitored by the imaging instruments on the Einstein Observatory, we have examined X-ray luminosity variations on the $10 \mathrm{yr}$ timescale between Einstein and $\operatorname{ROSAT}$ and find that up to $40 \%$ of the late-type stars are X-ray variable. Since there is only marginal evidence for increased variability on decade-long timescales, the variability observed on long and short timescales may have a common physical origin.
\end{abstract}

Subject headings: open clusters and associations: individual (Pleiades) - stars: coronae — stars: late-type stars: rotation $-X$-rays: stars

\section{INTRODUCTION}

The Pleiades is an important laboratory for the study of main-sequence stellar evolution because of its youth and proximity. With an estimated cluster age $\sim 70$ million years, many of the intermediate- and low-mass dwarfs are still rapidly rotating, providing clues to the evolution of angular momentum and lithium depletion on the main sequence. Rapid rotators are also expected to be copious $\mathrm{X}$-ray emitters, and, at a distance of $\sim 127 \mathrm{pc}$, a large number of intermediate- and lowmass cluster members are detectable with X-ray telescopes. Consequently, the Pleiades was one of the primary open clusters observed by the Einstein Observatory (Caillault \& Helfand 1985; Micela et al. 1990) and ROSAT (Stauffer et al. 1994; Micela et al. 1995). The analyses presented here represent a continuation of the Stauffer et al. (1994, hereafter Paper I) work. In that study, more than $300 \mathrm{X}$-ray sources were detected, of which 171 are associated with certain or probable cluster members. We follow up that spatial analysis with spectral and temporal analyses of X-ray sources associated with the late-type Pleiades stars.

The study of stellar X-ray spectra has been difficult because of the relatively poor energy resolution of the Einstein imaging

\footnotetext{
1 Department of Physics and Astronomy, University of Georgia, Athens, GA 30602-2451.

2 Joint Institute for Laboratory Astrophysics, University of Colorado, and National Institute of Standards and Technology, Boulder, CO 80309-0440.

${ }^{3}$ Harvard-Smithsonian Center for Astrophysics, 60 Garden Street, Cambridge, MA 02138
}

proportional counter (IPC) and the ROSAT position-sensitive proportional counter (PSPC). Nevertheless, many insights have been gained. For example, Schmitt et al. (1990), in a comprehensive study of IPC pulse-height spectra of late-type field stars, found that one- and two-temperature Raymond-Smith (1977, hereafter RS) optically thin plasma models fitted to the observed spectra were largely influenced by the signal-to-noise ratio of the data and that continuous emission measure distribution models were equally adequate and, perhaps, more physically meaningful. Schmitt et al. (1990) also found that $\mathbf{M}$ dwarfs display evidence of possessing high-temperature gas $\left(T>10^{7} \mathrm{~K}\right)$ in addition to lower temperature $\left(T \sim 3 \times 10^{6} \mathrm{~K}\right)$ material; the $F$ and $G$ dwarfs seemed to have only the low- $T$ component.

In a study of the Hyades stars, Stern et al. (1994) found that isothermal coronal plasma models do not fit the observed PSPC pulse-height spectra of the 10 brightest sources in the cluster (the only ones for which fits were attempted). Fits with models having two temperature components suggest that the higher of the two temperature components of the $\mathbf{M}$ dwarf coronae are generally hotter than those of the $F-G$ dwarf coronae, similar to the results found by Schmitt et al. (1990). Stern et al.'s interpretation of their results is that increased volumetric coronal heating, either in a steady fashion or in quasi-continuous flaring, may be producing the trend seen along the main sequence. They warn, though, that both their sample and that of Schmitt et al. are biased toward the high end of the $\mathrm{X}$-ray luminosity function.

Although the stars in the Pleiades are intrinsically more 
$X$-ray luminous than those in the Hyades, the fact that they are 3 times more distant means that their $\mathrm{X}$-ray fluxes are similar and, since the ROSAT PSPC exposure times for both clusters were approximately equal, the total counts observed for a typical individual star are approximately the same. Hence, as in the case of the Hyades, only a handful of Pleiades stars have sufficient counts with which to perform model fits to their $\mathrm{X}$-ray spectra. As in the Hyades, this means we are only probing the most X-ray-luminous sources. In order to include fainter Pleiades members, we have constructed composite $X$-ray spectra for different spectral types as a function of projected rotational velocity (see $\S 2$ below). In this manner, we hope to be able to determine in a statistically significant manner whether the coronal temperatures are dependent on spectral type (as implied by the previous studies) and/or on rotation (in some as yet to be determined manner). In addition, we can confirm (or refute) the expectation (Schmitt 1994) that the temperatures of the model components increase during a flare, since we observed one large flare from a star (H II 2147) for which its quiescent state is also sufficiently active to acquire a statistically valid model fit to its $X$-ray spectrum.

Preliminary temporal analyses of Pleiades $X$-ray data have been made by Schmitt et al. (1993), Stauffer et al. (1994), and Micela et al. (1995). Schmitt et al. (1993) compared ROSAT all-sky survey data with IPC data and suggested that the observed source variability in the decade between Einstein and ROSAT may be evidence for solar-like coronal activity cycles. In Paper I we treated the variability in only a cursory manner, determining that, as an ensemble, there is not great variation (less than a factor of $\sim 4$ ) in the $X$-ray emission from these stars over the decade between observations. Micela et al. (1995) find that $\sim 90 \%$ of the Pleiades stars are not variable (within a factor of $\sim 2$ ) on a timescale of approximately 6 months; they also find three stars which flared during their PSPC observation.

Hence, in addition to the spectral analysis, we have followed statistical techniques described by Schmitt et al. (1993), Gagné \& Caillault (1994), and Gagné, Caillault, \& Stauffer (1995) for measuring the variability of $X$-ray emission over many different timescales, ranging from minutes to more than a decade. We compare the medium-term variability (timescales of $\sim 1 \mathrm{yr}$ ) of the Pleiades stars with that of younger stars (e.g., those in Orion or $\rho \mathrm{Oph}$ ) and older stars (e.g., those in the Hyades), in terms of both frequency and strength of variation. By comparing the ROSAT PSPC and Einstein IPC count rates, we determine whether signatures of long-term solar-like dynamo activity cycles can be observed among the Pleiades stars.

\section{PSPC SPECTRAL ANALYSIS}

The ROSAT PSPC data for the core of the Pleiades cluster used in this paper have been described in detail in Paper I. The data consist of three pointed observations with individual exposure times ranging from 20.5 to $27.3 \mathrm{ks}$ and covering a total of $\sim 4.5 \mathrm{deg}^{2}$. A total of $317 \mathrm{X}$-ray sources were detected in this area, 171 of which have been associated with known or probable Pleiades cluster members.

The ROSAT X-ray telescope and PSPC have been described in detail by Pfeffermann et al. (1986) and Trümper (1992), respectively. The limited energy resolution $(E / \Delta E \sim 2.5$ at 1 $\mathrm{keV})$ of the PSPC has allowed us to construct backgroundsubtracted pulse-height spectra of the brighter (net counts $\geq 500$ ) sources. In addition, for faint and bright sources associated with late-type Pleiades members, we have combined the net counts according to spectral type and rotational velocity, thereby allowing us to look for differences in the PSPC spectra of rapidly and slowly rotating $F, G, K$, and $M$ stars.

\subsection{PSPC Spectra of Individual Cluster Stars}

Of the $171 \mathrm{X}$-ray sources associated with a single Pleiades cluster member, 15 were detected with 500 or more net counts in at least one of the three PSPC exposures. In the case of the bright X-ray flare star H II 2147 (see $\$ 3$ ), sufficient counts have been collected in and out of flare to construct both flare and quiescent spectra.

Counts have been extracted from $90 \%$ power-radius source circles and from concentric background annuli. The local background subtraction procedure we used is described in detail in Paper I. In particular, the background regions have been masked from nearby sources and the effective exposure times for each source have been estimated from broadband PSPC exposure maps.

In some cases a source has been detected in two exposures. In order to improve the signal-to-noise ratio of the $\mathrm{X}$-ray spectra of these stars, the counts in both the source and the background regions from the two exposures have been coadded and net rms errors have been determined. Since the energy response of the PSPC depends on the source's position on the detector, the net energy response of the detector for the co-added spectrum was determined by taking a weighted mean of the individual responses.

The resulting PSPC pulse-height spectra were fitted with one- and two-temperature RS models within PROS. (The PROS software is provided by the Harvard-Smithsonian Center for Astrophysics.) The hydrogen column density was fixed at $2 \times 10^{20} \mathrm{~cm}^{-2}$, consistent with the mean color excess $E(B-V) \sim 0.04$ found in the core of the cluster (see Paper I and references therein). When allowed to float, the best-fit column densities were always in the range $(1.6-2.5) \times 10^{20}$ $\mathrm{cm}^{-2}$, thereby supporting our assumption of approximately constant $N_{\mathrm{H}}$, at least for the purpose of fitting PSPC pulseheight spectra. In all cases, we find acceptable fits assuming cosmic abundances. That is not to say, however, that these coronae have cosmic abundances. The energy resolution and signal-to-noise ratios of the PSPC pulse-height spectra of the Pleiades stars are simply too poor to constrain coronal abundance values.

As was found by Schmitt et al. (1990) for stars detected with the Einstein IPC, we find that single-temperature models adequately fit the observed spectra when the total counts in the spectrum are low. For example, for five of six stars with fewer than 1000 net counts, a single-temperature model provides an acceptable fit, i.e., $\chi_{v}^{2} \leq 1$. This, of course, is an artifact of the $\chi^{2}$ statistic and indicates that the low-count spectra cannot constrain multitemperature models because of the large relative errors in each spectral bin. For all sources with net counts above 1000, two-temperature RS models were required.

In Table 1 we present the results of the RS model fits to the 15 sources with $\geq 500$ net counts associated with a known Pleiades cluster member. In column (1) we list the X-ray source number from Paper I; in columns (2)-(4) we list the name, $(B-V)_{0}$ color, and rotational velocity of the Pleiades stars. The remaining columns list the results of the spectral fits. In columns (5)-(10) we list the total number of net counts in the $0.1-2.0 \mathrm{keV}$ band, the best-fit values of $\chi_{v}^{2}$, the volume emission measure of the first temperature component $\left(E M_{1}\right)$, the temperature of the first component $\left(k T_{1}\right), E M_{2}$, and $k T_{2}$, respec- 


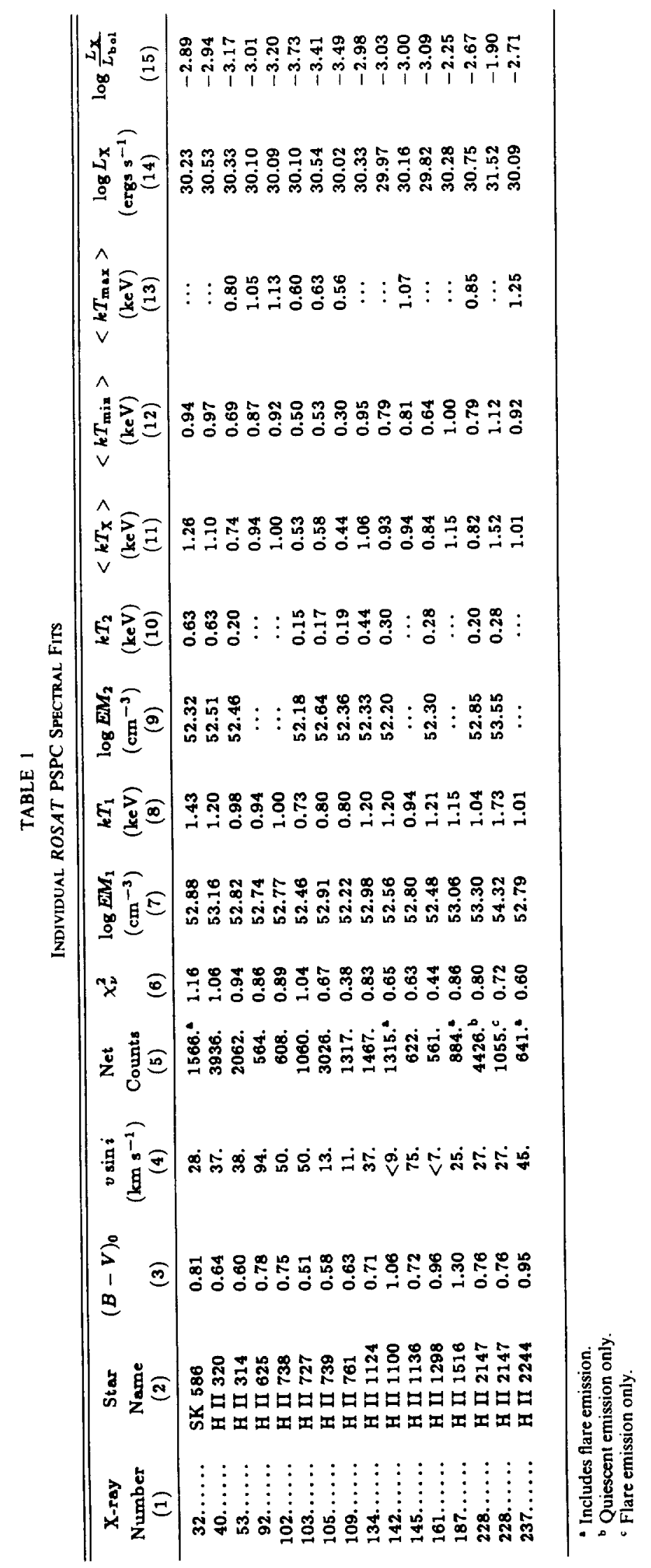


tively. Stars with no data in columns (9) and (10) were well fitted with a single-temperature model. We note that the PSPC pulse-height spectra have been corrected for vignetting, the off-axis decrease in telescope sensitivity.

The parameters in Table 1 are not meant to indicate absolute coronal temperature distributions. We use a twocomponent temperature distribution simply because these PSPC data cannot constrain more sophisticated models. In particular, continuous emission measure distributions do not provide better fits to the data. The best-fit temperatures and volume emission measures in Table 1 should, however, allow us to compare the relative distributions of coronal plasma among the different stars observed by ROSAT. As expected, during the H II 2147 flare we observe higher temperatures and much larger volume emission measures than during quiescence.

In Figure 1 we plot $k T_{2}$ against $k T_{1}$ for the 10 stars in Table 1 that have been fitted with the two-temperature model, including H II 2147 in and out of flare. The X-ray flare stars noted in Table 1 are indicated with a cross. The rapid rotators ( $v \sin i>16 \mathrm{~km} \mathrm{~s}^{-1}$ ) are indicated with filled symbols, while the slow rotators are indicated with open symbols. From Figure 1 we see that all three of the stars on which flares were seen have a dominant temperature component $k T_{1} \gtrsim 1 \mathrm{keV}$. We also note that three of the four stars with $k T_{1} \leqq 1 \mathrm{keV}$ are $F$ stars, while the fourth is a slowly rotating $G$ star. All stars with $k T_{1} \gtrsim 1 \mathrm{keV}$ are rapidly rotating $\mathrm{G}$ stars or $\mathrm{K}$ stars. No $M$ stars had sufficient counts to constrain a two-temperature model.

These results are in general agreement with those of Schmitt et al. (1990), who found generally hotter temperatures among $K$ - and $M$-type dwarfs than among $F$ - and G-type dwarfs observed by the Einstein IPC. We point out that their $\mathrm{G}$ star sample contained no stars with $v \sin i \gtrsim 16 \mathrm{~km} \mathrm{~s}^{-1}$. That is, the G stars with "hot" coronae in the Pleiades are rotating much more rapidly than the $\mathrm{G}$ stars examined by Schmitt et al. (1990). Since most field $G$ dwarfs are substantially older than their $70 \mathrm{Myr}$ old counterparts in the Pleiades, the dearth of rapidly rotating field $G$ dwarfs is not at all surprising given the timescales for stellar spin-down.

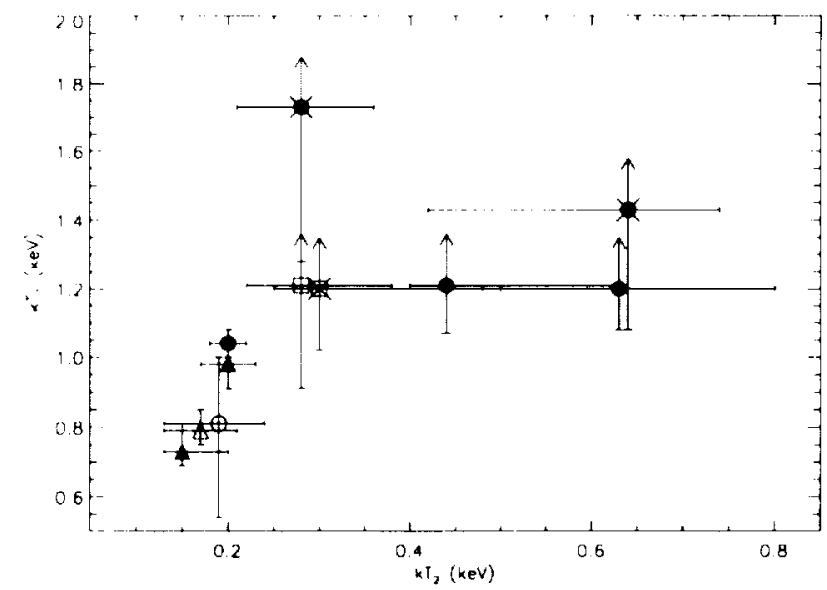

FIG. 1.-Coronal temperatures of $X$-ray-bright late-type Pleiades stars based on RS two-temperature model fits. Plotted are $k T_{1}$ and $k T_{2}(\mathrm{keV})$ for $F$ stars (triangles), $\mathrm{G}$ stars (circles), and $\mathrm{K}$ stars (squares), with open and filled symbols representing slow $\left(v \sin i \leq 16 \mathrm{~km} \mathrm{~s}^{-1}\right.$ ) and fast rotators, respectively. The error bars indicate $1 \sigma$ uncertainties in $k T_{1}$ and $k T_{2}$.
In order to compare results for one- and two-temperature model fits, we introduce a mean temperature,

$$
\left\langle T_{\mathbf{x}}\right\rangle=\frac{\sum_{i=1}^{N} T_{i} \mathrm{EM}_{i}}{\sum_{i=1}^{N} \mathrm{EM}_{i}},
$$

where $N=1,2$ for one-temperature and two-temperature model results, respectively.

For single-temperature models, $1 \sigma$ upper and lower bounds were determined by fixing $T$ and fitting EM for 0.1 $\mathrm{keV}<k T<2.0 \mathrm{keV}$ with $1 \sigma(68 \%)$ confidence corresponding to $\chi^{2}-\chi_{\min }^{2}=1.0$. In order to determine confidence contours for the two-temperature models, a best-fit $\chi^{2}$ value was computed at each point in the $k T_{1}$ versus $k T_{2}$ plane by fitting the two remaining free parameters $\left(E M_{1}\right.$ and $\left.E M_{2}\right)$. For two "interesting" parameters $\left(k T_{1}\right.$ and $\left.k T_{2}\right), 1 \sigma(68 \%)$ confidence corresponds to $\chi^{2}-\chi_{\min }^{2}=2.3$ (Lampton, Margon, \& Bowyer 1976; Avni 1976). Using equation (1) and the $1 \sigma$ minima and maxima of each temperature component, we have estimated the $1 \sigma$ minima and maxima of $\left\langle k T_{\mathbf{x}}\right\rangle$.

In columns (11)-(13) of Table 1 we list $\left\langle k T_{\mathbf{x}}\right\rangle,\left\langle k T_{\min }\right\rangle$, and $\left\langle k T_{\max }\right\rangle$. Stars with no data in column (13) indicate that the upper bound is not constrained by the data. Also indicated in columns (14) and (15) are $L_{\mathrm{X}}$ and $L_{\mathrm{X}} / L_{\text {bol }} . L_{\mathrm{X}}$ has been computed by integrating the best-fit RS incident spectrum and assuming a cluster distance of $127 \mathrm{pc}$ (Paper I) and a hydrogen column density of $2 \times 10^{20} \mathrm{~cm}^{-2}$. $L_{\text {bol }}$ has been inferred from Table 7 of Paper I.

We note that the $\mathrm{X}$-ray luminosities have been determined using the best-fit incident spectrum and not by assuming a constant count-rate conversion factor, as was done in Paper $I$. Consequently, the $L_{\mathbf{X}}$ values in Table 1 are slightly different from those in Table 7 of Paper 1 .

In Figure 2 we plot $\left\langle k T_{\mathbf{X}}\right\rangle$ versus $L_{\mathbf{X}} / L_{\text {bol }}$ for the stars in Table 1. We use $L_{\mathbf{X}} / L_{\text {bol }}$ as a measure of X-ray activity in these late-type stars because this ratio allows us to estimate what fraction of the star's total energy output is radiated in coronal soft X-rays.

The most striking trend seen in Figure 2 and in Table 1 is a general increase in mean coronal temperature with $X$-ray activity. While the sample of stars for which $\mathrm{X}$-ray spectra have been fitted is small and biased toward the most luminous stars,

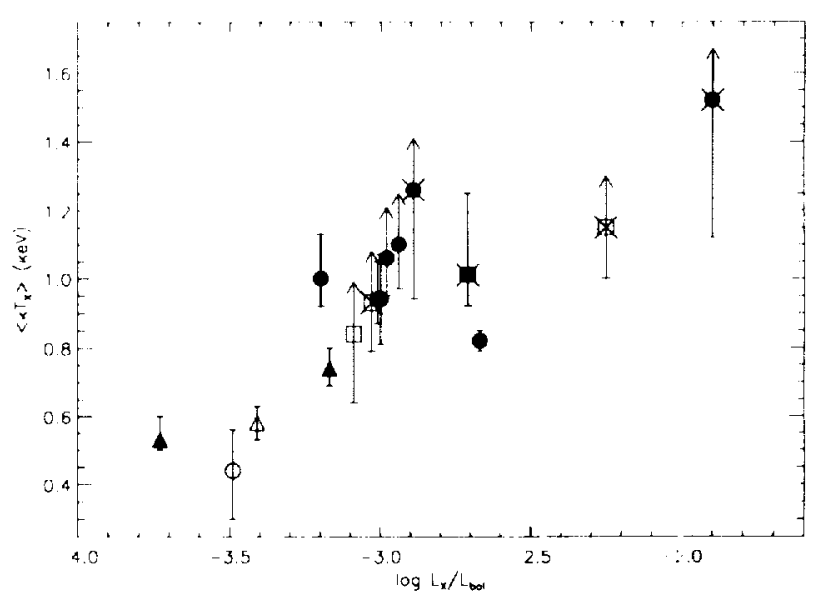

FiG. 2.-Mean coronal temperature vs. X-ray activity based on RS oneand two-temperature model fits. Plotted are $\left\langle k T_{\mathrm{X}}\right\rangle(\mathrm{keV})$ vs. $\log \left(L_{\mathrm{x}} / L_{\text {bo }}\right)$. See Fig. 1 for symbol definitions. 
this result, if correct, suggests that the coronae of the most active dwarfs do not simply contain more X-ray-emitting plasma than less active dwarfs, but that they contain generally hotter material as well. Again, a similar trend was noticed among late-type dwarfs, active binaries, and giants by Schmitt et al. (1990).

As was demonstrated in Paper I, rotation is the dominant determinant of X-ray activity in the late-type Pleiades stars. Figure 1 suggests that lower effective temperature and higher rotational velocity may lead to higher coronal temperatures. From Figure 2, the trend is for increasing temperatures with increasing $L_{\mathbf{X}} / L_{\text {bol. }}$. If $L_{\mathbf{x}} / L_{\text {bol }}$ is a surrogate measure of the coronal heating efficiency, then we interpret Figure 2 as an increase in plasma temperature with increased heating. However, the small number of stars we have analyzed do not allow us to correlate coronal temperature directly with rotational velocity.

Jordan \& Montesinos (1991) have examined correlations between coronal emission measure, coronal temperature, and physical stellar parameters for a number of late-type dwarfs and giants detected by Einstein. Their results point to higher coronal temperatures with increasing activity and decreasing Rossby number. The coronal temperatures and volume emission measures in Table 1 , in fact, obey the same scaling laws. See Figure 3 of Jordan \& Montesinos (1991).

\subsection{Composite PSPC Spectra of Cluster Stars}

While the results of the individual spectral fits may be suggestive, the few PSPC pulse-height spectra with sufficient counts to be well constrained are biased toward the most $X$ ray-luminous stars. In order to sample all the $\mathrm{X}$-ray-emitting late-type Pleiades stars, we have generated composite spectra by co-adding spectra of individual stars according to spectral type and rotational velocity. Total source and background counts for all sources in each group were collected for each of the three exposures. The spectra were co-added by simply summing net counts and determining net rms errors in each energy channel. Since counts from individual sources have been extracted from different $\mathrm{X}$-ray images and, hence, from different portions of the PSPC detector, the net energy response of the X-ray mirror assembly plus PSPC for the coadded spectrum has been calculated by determining a weighted mean of the individual energy responses.

Since the composite spectrum is a simple sum of individual spectra of different sources, the composite spectrum may be dominated by counts from a handful of stronger sources. While this may bias results toward the stronger sources, an alternative procedure (e.g., co-adding normalized spectra) would introduce larger net errors in the co-added spectrum, thereby poorly constraining the model parameters.

The stars used in these composite spectra are the late-type cluster members [those with $(B-V)_{0} \geq 0.35$ ] in Table 7 of Paper I. In Table 2 we present a synopsis of the star samples used to generate the composite spectra. In column (1) we group the Pleiades stars according to spectral type and rotational velocity, and in column (2) we list the total number of stars in each group. As before, we define the rapid rotators as those stars with $v$ vin $i>16 \mathrm{~km} \mathrm{~s}^{-1}$. In column (3) we list the total net counts in the $0.1-2.0 \mathrm{keV}$ passband, and in columns (4)-(7) we list the best-fit $\chi_{v}^{2}, k T_{1}, \log \left(E M_{1} / E M_{2}\right)$, and $k T_{2}$, respectively, from the RS two-temperature model fits.

As can be seen in column (3) of Table 2, for all but the slowly rotating $F$ stars, $\geq 1000$ net counts have been collected in each composite spectrum, allowing relatively tight constraints to be placed on the model parameters. By the same procedure described in $\$ 2.1, \chi^{2}$ confidence contours were determined for the composite spectra in Table 2 .

In Figure 3 we present the $\chi^{2} 68 \%$ and $90 \%$ confidence contours in the $k T_{1}$ versus $k T_{2}$ plane for all the $F$-type and $\mathrm{K}$-type cluster members. Also plotted are the $\chi^{2}$ contours for rapidly and slowly rotating $G$ stars. We have omitted the $M$ stars for the sake of clarity. In actuality, the $\mathbf{M}$-star contours are very close to those of the $K$ stars. The crosses indicate the best-fit temperatures. While, the F-, K- and M-type composite spectra can be adequately fitted without regard to rotation, the spectrum made up of all $G$ stars cannot be adequately fitted

TABLE 2

COMPOSITE ROSAT PSPC SPECTRAL FITS

\begin{tabular}{|c|c|c|c|c|c|c|}
\hline $\begin{array}{l}\text { Spectral } \\
\text { Group } \\
(1)\end{array}$ & $\begin{array}{c}\text { Number } \\
\text { of Stars } \\
(2)\end{array}$ & $\begin{array}{c}\text { Net } \\
\text { Counts } \\
(3)\end{array}$ & $\begin{array}{l}x_{\nu}^{2} \\
(4)\end{array}$ & $\begin{array}{c}k T_{1} \\
(\mathrm{keV}) \\
(5)\end{array}$ & $\begin{array}{c}\log E M_{2} / E M_{1} \\
(6)\end{array}$ & $\begin{array}{c}k T_{2} \\
(\mathrm{keV}) \\
(7)\end{array}$ \\
\hline \multicolumn{7}{|l|}{ F sters } \\
\hline $\begin{array}{l}\text { slow...... } \\
\text { fast...... } \\
\text { all..... }\end{array}$ & $\begin{array}{c}4 \\
6 \\
16 \\
\end{array}$ & $\begin{array}{r}601 \\
2668 . \\
6381 \\
\end{array}$ & $\begin{array}{l}\ldots \\
1.33 \\
0.91 \\
\end{array}$ & $\begin{array}{l}\ldots \\
0.71 \\
0.69 \\
\end{array}$ & $\begin{array}{r}\cdots \\
-0.26 \\
-0.22 \\
\end{array}$ & $\begin{array}{l}\cdots \\
0.15 \\
0.17 \\
\end{array}$ \\
\hline \multicolumn{7}{|l|}{ G stars } \\
\hline $\begin{array}{l}\text { slow...... } \\
\text { fast...... } \\
\text { all..... }\end{array}$ & $\begin{array}{l}21 \\
12 \\
33 \\
\end{array}$ & $\begin{array}{r}7890 . \\
14551 . \\
22441 . \\
\end{array}$ & $\begin{array}{l}0.98 \\
1.03 \\
1.83 \\
\end{array}$ & $\begin{array}{c}0.92 \\
1.27 \\
\ldots \\
\end{array}$ & $\begin{array}{c}-0.33 \\
-0.67 \\
\ldots \\
\end{array}$ & $\begin{array}{l}0.23 \\
0.52 \\
\cdots \\
\end{array}$ \\
\hline \multicolumn{7}{|l|}{ K stars } \\
\hline $\begin{array}{l}\text { slow...... } \\
\text { fost....... } \\
\text { all..... }\end{array}$ & $\begin{array}{l}24 \\
16 \\
47 \\
\end{array}$ & $\begin{array}{r}5782 . \\
7548 . \\
13966 . \\
\end{array}$ & $\begin{array}{l}1.26 \\
1.14 \\
1.35 \\
\end{array}$ & $\begin{array}{l}1.14 \\
1.08 \\
1.11 \\
\end{array}$ & $\begin{array}{l}-0.28 \\
-0.30 \\
-0.29 \\
\end{array}$ & $\begin{array}{l}0.30 \\
0.26 \\
0.28 \\
\end{array}$ \\
\hline \multicolumn{7}{|l|}{ M stars } \\
\hline $\begin{array}{l}\text { slow...... } \\
\text { fast...... } \\
\text { all..... }\end{array}$ & $\begin{array}{c}6 \\
5 \\
47 \\
\end{array}$ & $\begin{array}{l}1233 . \\
1093 . \\
8678 .\end{array}$ & $\begin{array}{l}0.40 \\
0.68 \\
1.06 \\
\end{array}$ & $\begin{array}{l}0.95 \\
1.15 \\
1.11 \\
\end{array}$ & $\begin{array}{l}-0.04 \\
-0.26 \\
-0.31 \\
\end{array}$ & $\begin{array}{l}0.20 \\
0.35 \\
0.26 \\
\end{array}$ \\
\hline
\end{tabular}




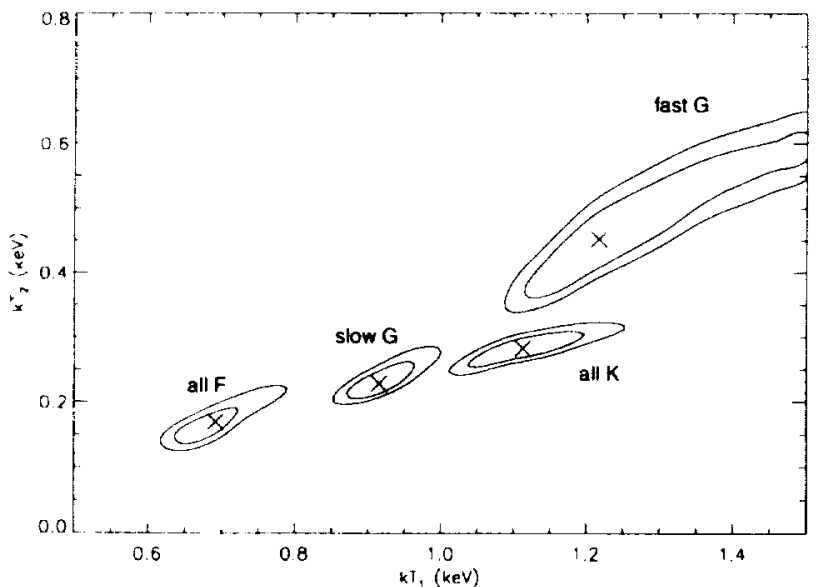

Fig. 3.-Coronal temperatures of all X-ray-detected late-type Pleiades stars based on RS two-temperature model fits of the composite spectra. Plotted are the $\chi^{2}$ contours in the $k T_{1}$ vs. $k T_{2}$ (keV) plane for $\mathrm{F}$ stars, slow $\mathrm{G}$ stars, fast $G$ stars, and $K$ stars. The $M$ star contours have not been indicated for the sake of clarity; they are very close to the $\mathrm{K}$ star contours. The inner and outer contour lines represent, respectively, the $1 \sigma$ and $1.6 \sigma(68 \%$ and $90 \%)$ confidence intervals in the fitted temperatures.

with a two-temperature model. On the other hand, when we separate the fast and slow G stars, we find acceptable fits with substantially hotter distributions for the rapid rotators. We note that at the $90 \%$ confidence level, the model temperatures from the $F, K$, and rapidly and slowly rotating $G$ stars do not overlap.

Results of the RS model fits to the individual and composite spectra both point to increased coronal temperatures with decreasing effective temperature. There is also a suggestion that rotation may play a role in determining coronal temperature distributions, at least among the handful of ROSATdetected $G$ stars. This apparent dependence on rotation is probably an artifact of the overall increase in activity with rotational velocity. That is, the G-type Pleiades stars with higher rotational velocities have higher $L_{\mathbf{X}} / L_{\text {bol }} \sim 10^{-3}$ and, consequently, hotter coronae.

\subsection{PSPC Hardness Ratios of Cluster Stars}

The coronal temperatures determined in the previous sections are model-dependent, with different models (e.g., one-

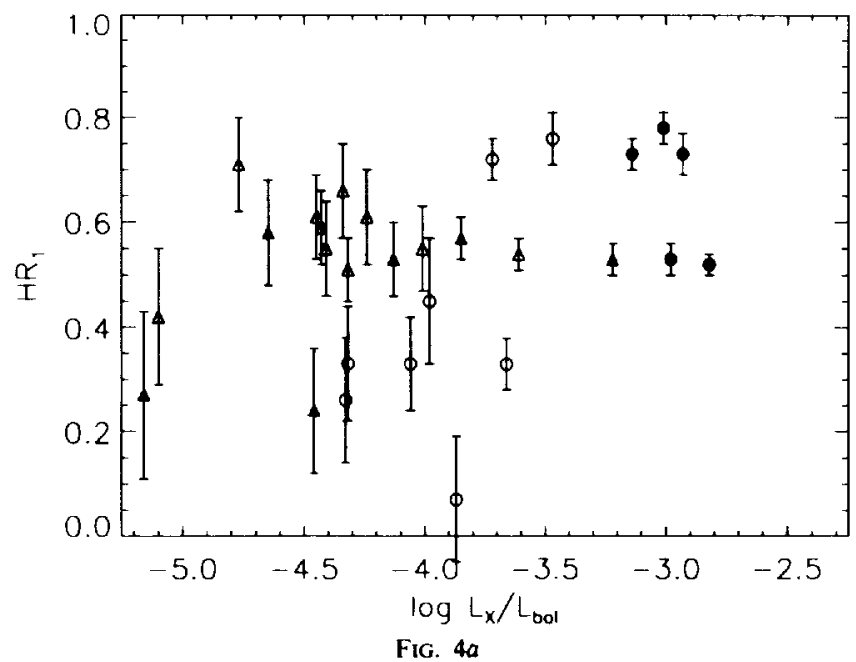

temperature, two-temperature, or continuous emission measure distribution), different coronal abundances, and different plasma codes providing different best-fit plasma distributions. An alternative approach to modeling the PSPC pulse-height spectra is to examine the hardness ratios of the detected sources. Analogous to optical photometric colours, $\mathrm{X}$-ray hardness ratios have been used to estimate the level of circumstellar and interstellar hydrogen absorption. In addition, accurate hardness ratio determinations can be used to estimate roughly the characteristic temperature of the emitting coronal plasma (e.g., see Fig. 4 of Neuhäuser et al. 1995b).

We use the standard $R O S A T$ soft, medium, and hard bands defined, respectively, as $s=$ net counts in channels $10-40(0.1-$ $0.28 \mathrm{keV}), m=$ net counts in channels $51-90(0.5-0.9 \mathrm{keV})$, and $h=$ net counts in channels $91-200(0.9-2.0 \mathrm{keV})$, and hardness ratios (Neuhäuser et al. 1995a) as

$$
\begin{aligned}
\mathbf{H R}_{1} & =\frac{h+m-s}{h+m+s}, \\
\mathrm{HR}_{2} & =\frac{h-m}{h+m} .
\end{aligned}
$$

We have determined $3 \sigma$ count rates or upper limits in the three PSPC passbands for the $146 \mathrm{X}$-ray sources associated with a single F-, G-, K-, or M-type Pleiades cluster member. These count rates have yielded $H_{R_{1}}$ and $H R_{2}$ values for 79 stars. Because of upper limits in the soft band, in 58 cases only $\mathrm{HR}_{2}$ could be determined. Because of upper limits in the medium or hard bands, in an additional nine cases neither $H_{1}$ nor $\mathrm{HR}_{2}$ could be determined. In Table 3 we present the hardness ratios and optical data for the 137 stars for which $\mathrm{HR}_{1}$ or $\mathrm{HR}_{2}$ could be determined. In columns (1)-(5) we list the $\mathrm{X}$-ray source number, star name, $(B-V)_{0},\left(V-\eta_{0}\right.$, and $v$ $\sin i$ from Table 7 of Paper I. In columns (6)-(9) we list $H_{R_{1}}$, $\sigma_{\mathrm{HR}_{1}}, \mathrm{HR}_{2}$ and $\sigma_{\mathrm{HR}_{2}}$. In columns (10) and (11) we list corresponding values of $L_{\mathrm{x}}$ and $L_{\mathrm{x}} / L_{\text {bol }}$ from Paper $\mathrm{I}$.

In Figures 4 and 5 we plot the resulting hardness ratios versus $L_{\mathrm{x}} / L_{\text {bol }}$. See Figure 1 for symbol definitions. Figures $4 a$ and $4 b$ do not suggest higher $H_{R_{1}}$ values with increased $L_{\mathbf{X}} / L_{\text {bol }}$. However, many of the $\mathrm{K}$ and $\mathrm{M}$ stars for which hardness ratios could be determined, i.e., the brightest ones, have values of $L_{\mathrm{x}} / L_{\mathrm{bol}}$ close to the saturation value of $10^{-3}$. The trend in Figure $5 a$ is for increasing hardness ratio $\mathrm{HR}_{2}$ with

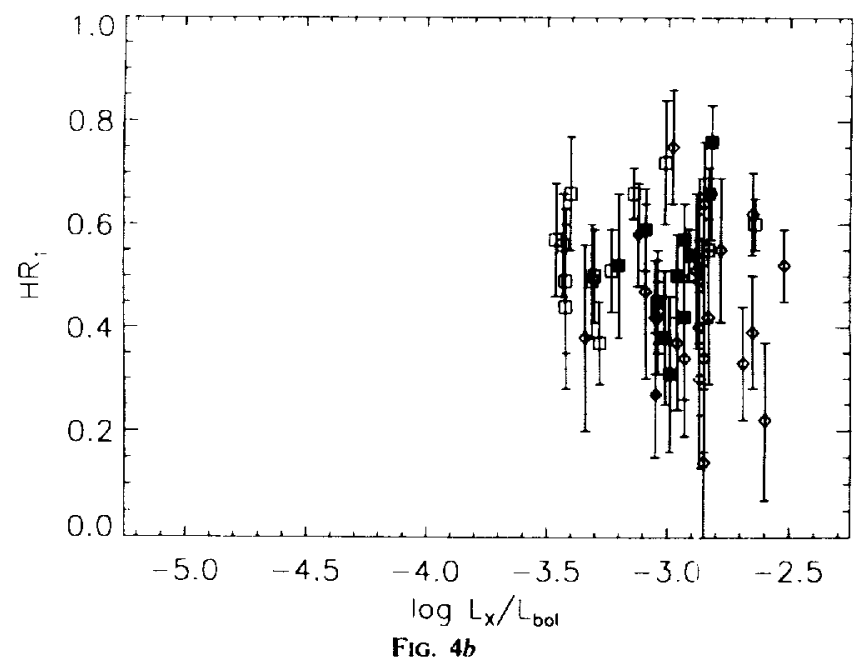

Fig. 4. - HR 1 vs. $L_{\mathrm{x}}$ for (a) F stars (trangles) and $\mathrm{G}$ stars (circles) and (b) $\mathrm{K}$ stars (squares) and $\mathrm{M}$ stars (diamonds). Open and filled symbols represent slow ( $v$ sin $\left.i \leq 16 \mathrm{~km} \mathrm{~s}^{-1}\right)$ and fast rotators, respectively. 
TABLE 3

ROSAT PSPC HARDNESS RATIOS

\begin{tabular}{|c|c|c|c|c|c|c|c|c|c|c|}
\hline $\begin{array}{c}\text { X-ray } \\
\text { Number } \\
(1)\end{array}$ & $\begin{array}{c}\text { Star } \\
\text { Name } \\
\text { (2) }\end{array}$ & $\begin{array}{c}(B-V)_{0} \\
(3)\end{array}$ & $(V-I)_{0}$ & $\begin{array}{c}v \sin i \\
\left(\mathrm{~km} \mathrm{~s}^{-1}\right) \\
(5)\end{array}$ & $\begin{array}{l}H R_{1} \\
(6)\end{array}$ & $\begin{array}{l}\sigma_{1} \\
(7)\end{array}$ & $\begin{array}{r}H R_{2} \\
(8)\end{array}$ & $\begin{array}{l}\sigma_{2} \\
(9)\end{array}$ & $\begin{array}{c}L_{X} \\
\left(\operatorname{ergs}^{-1}\right) \\
(10)\end{array}$ & $\begin{array}{l}\frac{L_{X}}{L_{b 01}} \\
(11)\end{array}$ \\
\hline $2 \ldots \ldots$ & HCG 65 & 1.32 & 1.48 & $\ldots$ & $\cdots$ & $\cdots$ & -0.01 & 0.11 & 29.67 & -3.20 \\
\hline $4 . \ldots \ldots$ & HCG 71 & 1.37 & 1.68 & $\cdots$ & $\ldots$ & $\cdots$ & 0.22 & 0.18 & 29.21 & -3.21 \\
\hline $6 \ldots .$. & H II 25 & 0.44 & $\ldots$ & $\cdots$ & $\cdots$ & $\cdots$ & -0.27 & 0.17 & 29.02 & -4.89 \\
\hline $8 . \ldots$. & H II 34 & 0.88 & 0.71 & $<7$ & $\cdots$ & $\ldots$ & 0.16 & 0.20 & 28.98 & -4.04 \\
\hline o...... & HCG 97 & $\ldots$ & 2.02 & $\cdots$ & $\cdots$ & $\ldots$ & 0.16 & 0.08 & 29.42 & -2.88 \\
\hline $10 . \ldots \ldots$ & HCG 100 & 1.56 & 2.46 & 11. & 0.30 & 0.17 & -0.18 & 0.19 & 29.16 & -2.87 \\
\hline $12 \ldots \ldots$ & HCG 103 & $\cdots$ & 2.30 & $\cdots$ & .. & $\ldots$ & 0.07 & 0.20 & 28.82 & -3.26 \\
\hline $17 \ldots \ldots$ & AK1B 121 & $\ldots$ & . & $\cdots$ & $\ldots$ & $\cdots$ & 0.00 & 0.06 & 29.95 & $-\mathbf{3 . 3 3}$ \\
\hline $18 \ldots \ldots$ & H II 97 & 1.04 & 0.99 & $<8$ & 0.57 & 0.11 & 0.08 & 0.09 & 29.39 & -3.46 \\
\hline $24 \ldots \ldots$ & H II 134 & 1.43 & 1.88 & 70. & $\ldots$ & $\ldots$ & 0.03 & 0.12 & 29.42 & -3.16 \\
\hline $25 \ldots \ldots$ & HCG 115 & $\ldots$ & 2.31 & $\cdots$ & $\cdots$ & $\ldots$ & -0.20 & 0.16 & 29.27 & -2.68 \\
\hline $26 . \ldots \ldots$ & H II 133 & 1.31 & 1.49 & 19. & $\cdots$ & $\cdots$ & -0.01 & 0.10 & 29.43 & -3.01 \\
\hline $30 . \ldots \ldots$ & HCG 123 & 1.53 & 2.10 & 13. & 0.37 & 0.13 & 0.06 & 0.14 & 29.29 & -2.96 \\
\hline $31 \ldots \ldots$ & H II 173 & 0.80 & 0.63 & $<7$ & .. & $\cdots$ & -0.10 & 0.19 & 29.16 & -4.25 \\
\hline $32 \ldots \ldots$ & H II 174 & 0.81 & .. & 28. & 0.73 & 0.04 & 0.13 & 0.03 & 30.19 & -2.93 \\
\hline $35 \ldots \ldots$ & H II 191 & 1.31 & 1.60 & $\ldots$ & 0.75 & 0.11 & 0.10 & 0.08 & 29.40 & -2.98 \\
\hline 36...... & H II 212 & 1.33 & 1.51 & 10. & 0.43 & 0.12 & 0.12 & 0.11 & 29.41 & -3.04 \\
\hline $37 \ldots \ldots$ & HCG 129 & $\cdots$ & 1.96 & $\ldots$ & $\ldots$ & .. & 0.27 & 0.19 & 28.85 & -3.11 \\
\hline $39 . \ldots \ldots$ & HCG 134 & 1.47 & 2.03 & $\ldots$ & 0.51 & 0.15 & 0.23 & 0.12 & 28.26 & -2.88 \\
\hline $40 . \ldots$ & H II 253 & 0.64 & $\ldots$ & 37. & 0.78 & 0.03 & 0.16 & 0.02 & 30.46 & $-\mathbf{3 . 0 1}$ \\
\hline $41 \ldots \ldots$ & H II 263 & 0.84 & 0.69 & 10. & .. & $\cdots$ & 0.05 & 0.05 & 29.80 & $-\mathbf{3 . 3 3}$ \\
\hline $42 \ldots \ldots$ & H II 250 & 0.63 & 0.45 & $<7$ & 0.33 & 0.09 & -0.11 & 0.10 & 29.39 & -4.06 \\
\hline $43 . \ldots \ldots$ & SK 586 & $\cdots$ & 2.54 & $\cdots$ & $\cdots$ & $\cdots$ & -0.09 & 0.23 & 28.95 & -2.83 \\
\hline $46 \ldots \ldots$ & H II 293 & 0.66 & 0.49 & $<7$ & $\cdots$ & $\cdots$ & -0.16 & 0.14 & 29.13 & -4.29 \\
\hline $49 . \ldots \ldots$ & H II 303 & 0.84 & 0.78 & 16. & 0.72 & 0.04 & 0.08 & 0.03 & 29.87 & -3.72 \\
\hline $53 \ldots \ldots$ & H II 314 & 0.60 & . & 38. & 0.53 & 0.03 & 0.09 & 0.03 & 30.28 & -3.22 \\
\hline $54 \ldots \ldots$ & H II 320 & 0.84 & $\cdots$ & 11. & 0.76 & 0.05 & 0.02 & 0.04 & 29.90 & -3.47 \\
\hline $55 \ldots \ldots$ & H II 324 & 1.01 & 1.03 & 72. & 0.66 & 0.05 & 0.06 & 0.04 & 29.85 & -2.83 \\
\hline $57 \ldots \ldots$ & H II 338 & 0.42 & $\cdots$ & $\cdots$ & 0.71 & 0.09 & -0.04 & 0.07 & 29.30 & -4.77 \\
\hline $58 \ldots \ldots$ & H II 335 & 1.22 & 1.40 & 73. & $\cdots$ & ... & 0.08 & 0.08 & 29.63 & -2.89 \\
\hline $59 . \ldots \ldots$ & HCG 149 & 1.51 & 1.97 & 20. & 0.66 & 0.09 & 0.20 & 0.08 & 29.38 & -2.82 \\
\hline $60 \ldots$. & HCG 148 & $\cdots$ & 3.04 & $\cdots$ & .. & $\ldots$ & 0.07 & 0.10 & 29.27 & -2.58 \\
\hline $62 \ldots \ldots$ & H II 345 & 0.80 & $\ldots$ & 18. & 0.53 & 0.03 & 0.07 & 0.03 & 30.16 & -2.98 \\
\hline $63 \ldots \ldots$ & HCG 150 & 1.47 & 2.47 & $\cdots$ & 0.39 & 0.11 & -0.07 & 0.12 & 29.27 & -2.65 \\
\hline $64 \ldots \ldots$ & HCG 156 & $\ldots$ & 2.29 & $\cdots$ & $\ldots$ & $\ldots$ & -0.05 & 0.26 & 28.79 & -3.28 \\
\hline $65 \ldots \ldots$ & H II 347 & 1.37 & 1.70 & 75. & 0.58 & 0.10 & 0.03 & 0.08 & 29.54 & $-\mathbf{3 . 1 2}$ \\
\hline $66 . \ldots \ldots$ & H II 357 & 1.17 & 1.27 & $<9$. & 0.55 & 0.14 & -0.06 & 0.11 & 29.83 & -2.83 \\
\hline $69 \ldots \ldots$ & H II 405 & 0.50 & $\ldots$ & 17. & 0.53 & 0.07 & -0.14 & 0.06 & 29.65 & -4.13 \\
\hline $71 \ldots \ldots$ & H II 451 & 1.17 & 1.18 & $<9$. & 0.49 & 0.14 & -0.11 & 0.12 & 29.19 & -3.42 \\
\hline $72 \ldots \ldots$ & H II 476 & 0.52 & 0.22 & 23. & $\ldots$ & $\cdots$ & 0.22 & 0.14 & 29.52 & -4.17 \\
\hline $74 \ldots \ldots$ & H II 489 & 0.58 & 0.44 & 17. & $\cdots$ & $\cdots$ & -0.17 & 0.11 & 29.32 & -4.24 \\
\hline $79 . \ldots$ & HCG 172 & .. & 2.95 & $\cdots$ & $\cdots$ & $\cdots$ & -0.05 & 0.22 & 28.86 & -2.62 \\
\hline $80 \ldots \ldots$ & H II 514 & 0.66 & 0.48 & 11. & $\ldots$ & $\cdots$ & -0.24 & 0.14 & 29.21 & -4.22 \\
\hline $85 \ldots \ldots$ & H II 554 & 1.28 & 1.42 & $\ldots$ & 0.49 & 0.11 & -0.05 & 0.10 & 29.26 & $-\mathbf{3 . 3 1}$ \\
\hline $89 \ldots \ldots$ & HCG 178 & 1.61 & 2.50 & 45. & .. & .. & -0.19 & 0.13 & 29.34 & -2.77 \\
\hline $90 . \ldots \ldots$ & HCG 181 & 1.53 & 2.28 & $\cdots$ & 0.52 & 0.07 & 0.08 & 0.06 & 29.50 & -2.52 \\
\hline $92 \ldots \ldots$ & H II 625 & 0.78 & 0.55 & 94. & .. & $\ldots$ & 0.23 & 0.05 & 30.19 & -2.92 \\
\hline $93 . \ldots \ldots$ & H II 605 & 0.40 & $\ldots$ & 80. & 0.27 & 0.16 & -0.16 & 0.16 & 28.94 & -5.16 \\
\hline $94 \ldots \ldots$ & H II 624 & 1.49 & 1.92 & 16. & $\cdots$ & $\cdots$ & -0.24 & 0.17 & 28.03 & -3.20 \\
\hline $95 \ldots \ldots$ & H II 627 & 0.46 & $\ldots$ & ... & 0.66 & 0.09 & -0.23 & 0.08 & 29.49 & -4.34 \\
\hline $97 \ldots \ldots$ & H II 686 & 1.39 & 1.32 & 68. & 0.57 & 0.07 & -0.09 & 0.06 & 29.75 & -2.93 \\
\hline $98 \ldots$. & H II 708 & 0.58 & $\cdots$ & 48. & $\ldots$ & $\ldots$ & 0.03 & 0.05 & 29.78 & -3.89 \\
\hline $100 \ldots \ldots$ & HCG 194 & $\cdots$ & 2.37 & $\cdots$ & $\cdots$ & $\cdots$ & 0.01 & 0.11 & 29.20 & -2.71 \\
\hline $102 \ldots \ldots$ & H II 738 & 0.75 & 0.56 & 50. & $\cdots$ & $\cdots$ & 0.32 & 0.05 & 30.25 & -3.04 \\
\hline $103 \ldots \ldots$ & H II 727 & 0.51 & $\ldots$ & 50. & 0.57 & 0.04 & -0.11 & 0.04 & 29.98 & -3.85 \\
\hline $104 \ldots \ldots$ & H II 745 & 0.48 & $\cdots$ & $\cdots$ & 0.55 & 0.09 & 0.04 & 0.08 & 29.52 & -4.41 \\
\hline $105 \ldots \ldots$ & H II 739 & 0.58 & 0.44 & 13. & 0.54 & 0.03 & 0.00 & 0.02 & 30.29 & -3.61 \\
\hline $107 \ldots \ldots$ & H II 746 & 0.73 & 0.61 & $<7$ & $\ldots$ & $\cdots$ & -0.04 & 0.11 & 29.06 & -4.17 \\
\hline
\end{tabular}


TABLE 3-Continued

\begin{tabular}{|c|c|c|c|c|c|c|c|c|c|c|}
\hline $\begin{array}{c}\text { X-ray } \\
\text { Nurnber } \\
\text { (1) }\end{array}$ & $\begin{array}{c}\text { Star } \\
\text { Name } \\
(2)\end{array}$ & $\begin{array}{c}(B-V)_{0} \\
(3)\end{array}$ & $\begin{array}{c}(V-I)_{0} \\
(4)\end{array}$ & $\begin{array}{c}v \sin i \\
\left(\mathrm{~km} \mathrm{~s} \mathrm{~s}^{-1}\right) \\
(5)\end{array}$ & $\begin{array}{l}H R_{1} \\
(6)\end{array}$ & (7) & $\begin{array}{r}H R_{2} \\
(8)\end{array}$ & $\begin{array}{r}\sigma_{2} \\
(9)\end{array}$ & $\begin{array}{c}L_{\mathrm{X}} \\
\left(\text { ergs }^{-1}\right) \\
(10)\end{array}$ & $\begin{array}{l}\frac{L_{X}}{L_{b o l}} \\
(11)\end{array}$ \\
\hline 108. & H II 762 & 1.36 & 1.48 & $<8$ & $\cdots$ & $\ldots$ & 0.01 & 0.10 & 29.33 & -3.16 \\
\hline $109 \ldots \ldots$ & H II 761 & 0.63 & $\ldots$ & 11. & 0.33 & 0.05 & -0.11 & 0.05 & 29.85 & -3.66 \\
\hline $115 \ldots$. & SK 488 & $\ldots$ & 2.10 & $\ldots$ & 0.64 & 0.12 & 0.08 & 0.08 & 28.25 & -2.85 \\
\hline $116 . . .$. & H II 886 & 0.98 & 0.95 & $<8$ & $\cdots$ & $\cdots$ & 0.08 & 0.26 & 28.98 & -4.05 \\
\hline $117 \ldots .$. & H II 880 & 1.29 & 1.65 & $<9$. & 0.38 & 0.18 & 0.24 & 0.18 & 29.02 & -3.34 \\
\hline 119..... & H II 806 & 1.48 & 2.10 & $\ldots$ & 0.49 & 0.13 & -0.03 & 0.08 & 29.51 & -2.87 \\
\hline $120 \ldots .$. & H II 930 & 1.22 & 1.48 & 20. & 0.52 & 0.14 & -0.06 & 0.12 & 29.15 & -3.20 \\
\hline $121 \ldots \ldots$ & H II 916 & 0.83 & $\ldots$ & $<7$ & 0.07 & 0.12 & 0.08 & 0.12 & 29.22 & -3.87 \\
\hline $126 \ldots \ldots$ & H II 996 & 0.59 & 0.41 & 10. & 0.55 & 0.08 & 0.03 & 0.07 & 29.55 & -4.01 \\
\hline $130 \ldots \ldots$ & HCG 219 & 1.55 & 2.26 & $\ldots$ & 0.47 & 0.17 & 0.06 & 0.15 & 29.06 & -3.09 \\
\hline $132 \ldots \ldots$ & H II 1039 & 0.93 & 0.66 & $<7$ & $\ldots$ & $\ldots$ & 0.08 & 0.11 & 29.85 & -3.14 \\
\hline $134 \ldots \ldots$ & H II 1032 & 0.71 & 0.57 & 37. & 0.73 & 0.03 & 0.10 & 0.03 & 30.16 & -3.14 \\
\hline $136 . . .$. & H II 1061 & 1.15 & 1.23 & $<\theta$. & 0.72 & 0.12 & 0.14 & 0.09 & 29.51 & $-\mathbf{3 . 0 1}$ \\
\hline $141 \ldots$ & H II 1094 & 1.36 & 1.56 & $\ldots$ & $\ldots$ & $\cdots$ & 0.01 & 0.09 & 29.60 & -3.00 \\
\hline $142 \ldots \ldots$ & H II 1100 & 1.06 & 1.02 & $<\boldsymbol{\theta}$. & 0.66 & 0.05 & 0.07 & 0.04 & 29.87 & -3.14 \\
\hline $143 \ldots \ldots$ & H II 1117 & 0.69 & $\ldots$ & $<7$. & 0.26 & 0.12 & -0.02 & 0.13 & 29.33 & -4.33 \\
\hline $144 \ldots \ldots$ & H II 1122 & 0.42 & $\ldots$ & $\cdots$ & 0.59 & 0.07 & -0.18 & 0.08 & 29.55 & -4.43 \\
\hline $145 \ldots \ldots$ & H II 1136 & 0.72 & 0.57 & 75. & $\ldots$ & $\ldots$ & 0.08 & 0.07 & 30.14 & -3.02 \\
\hline $146 \ldots \ldots$ & H II 1124 & 0.94 & 0.81 & $<8$. & 0.56 & 0.10 & 0.06 & 0.08 & 29.47 & -3.43 \\
\hline $147 \ldots \ldots$ & H II 1101 & 0.57 & 0.42 & 18. & $\cdots$ & $\cdots$ & -0.27 & 0.18 & 29.37 & -4.24 \\
\hline $163 \ldots \ldots$ & H II 1215 & 0.58 & 0.42 & $<7$ & $\ldots$ & $\ldots$ & -0.20 & 0.16 & 28.34 & -4.16 \\
\hline $154 \ldots .$. & HCG 244 & $\cdots$ & 2.45 & $\ldots$ & $\ldots$ & $\ldots$ & -0.05 & 0.11 & 29.21 & -2.81 \\
\hline 156.. & HCG 246 & $\ldots$ & 2.67 & $\ldots$ & 0.22 & 0.15 & -0.01 & 0.17 & 29.11 & -2.60 \\
\hline $160 .$. & H II 1280 & 1.40 & 1.61 & 75. & 0.62 & 0.08 & 0.05 & 0.07 & 28.44 & -2.65 \\
\hline $161 \ldots \ldots$ & H II 1298 & 0.96 & 0.82 & $<7$ & 0.66 & 0.11 & -0.04 & 0.08 & 29.51 & -3.40 \\
\hline $164 . \ldots \ldots$ & H II $\mathbf{1 3 2 1}$ & 1.43 & 2.17 & 17. & $\ldots$ & $\ldots$ & -0.37 & 0.19 & 29.16 & -3.20 \\
\hline $167 \ldots \ldots$ & H II 1309 & 0.43 & $\cdots$ & $\ldots$ & 0.51 & 0.06 & -0.21 & 0.05 & 29.60 & -4.32 \\
\hline $168 \ldots \ldots$ & HCG 258 & 1.50 & 2.27 & $\cdots$ & $\ldots$ & $\ldots$ & -0.01 & 0.20 & 29.27 & -2.91 \\
\hline $169 \ldots \ldots$ & H II 1332 & 0.98 & 0.84 & $<8$. & 0.37 & 0.08 & -0.02 & 0.09 & 29.57 & -3.28 \\
\hline $170 \ldots \ldots$ & H II $\mathbf{1 3 3 8}$ & 0.42 & $\ldots$ & 10. & 0.42 & 0.13 & 0.00 & 0.12 & 29.12 & -5.10 \\
\hline $172 \ldots \ldots$ & H II 1365 & 1.36 & 1.65 & $<\theta$ & 0.42 & 0.07 & -0.03 & 0.07 & 29.59 & -3.04 \\
\hline $174 . \ldots$. & H II 1348 & 1.11 & 1.12 & $<\theta$. & $\ldots$ & $\ldots$ & -0.08 & 0.16 & 29.09 & $-\mathbf{3 . 7 7}$ \\
\hline $181 \ldots \ldots$ & H II 1464 & 1.04 & 0.98 & $<\theta$. & $\ldots$ & $\ldots$ & -0.20 & 0.21 & 29.16 & -3.59 \\
\hline $182 \ldots \ldots$ & HCG 273 & $\ldots$ & 1.86 & $\ldots$ & 0.42 & 0.13 & 0.06 & 0.10 & 29.26 & -2.83 \\
\hline $187 \ldots \ldots$ & H II 1516 & 1.30 & 1.43 & 25. & 0.60 & 0.05 & 0.11 & 0.05 & 28.89 & -2.64 \\
\hline $188 \ldots .$. & H II 1514 & 0.62 & 0.44 & 15. & 0.61 & 0.09 & 0.03 & 0.07 & 29.27 & -4.24 \\
\hline $189 \ldots \ldots$ & H II 1532 & 1.23 & 1.37 & 32. & 0.38 & 0.13 & -0.03 & 0.12 & 29.43 & -3.01 \\
\hline $190 \ldots \ldots$ & H II 1531 & 1.23 & 1.22 & 60. & 0.45 & 0.08 & 0.00 & 0.08 & 29.56 & -3.04 \\
\hline $195 \ldots \ldots$ & H II 1613 & 0.50 & $\cdots$ & 19. & 0.24 & 0.12 & -0.59 & 0.15 & 28.30 & -4.46 \\
\hline $187 \ldots \ldots$ & H II 1653 & 1.17 & 1.25 & 35. & 0.31 & 0.15 & 0.00 & 0.09 & 29.59 & -2.99 \\
\hline $188 \ldots \ldots$ & H II 1726 & 0.51 & $\cdots$ & 12. & 0.61 & 0.08 & -0.21 & 0.06 & 29.56 & -4.45 \\
\hline $210 \ldots \ldots$ & H II 1827 & 1.46 & 1.96 & $\cdots$ & $\cdots$ & $\cdots$ & -0.01 & 0.18 & 29.28 & -3.15 \\
\hline $211 \ldots \ldots$ & H II 1856 & 0.52 & $\ldots$ & 16. & $\ldots$ & $\ldots$ & -0.15 & 0.11 & 29.10 & -4.60 \\
\hline $217 \ldots \ldots$ & H II 1912 & 0.45 & $\ldots$ & 75. & 0.58 & 0.10 & 0.08 & 0.07 & 28.43 & -4.65 \\
\hline $219 \ldots \ldots$ & HCG 337 & $\ldots$ & 2.37 & $\ldots$ & $\ldots$ & $\ldots$ & -0.07 & 0.25 & 28.05 & -2.81 \\
\hline $222 \ldots \ldots$ & H II 2034 & 0.92 & 0.89 & 77. & 0.59 & 0.08 & -0.05 & 0.07 & 29.67 & -3.09 \\
\hline $223 \ldots \ldots$ & H II 2027 & 0.81 & 0.65 & $<8$. & 0.45 & 0.12 & 0.03 & 0.09 & 28.42 & -3.98 \\
\hline $224 \ldots \ldots$ & HCG 348 & $\ldots$ & 2.48 & $\ldots$ & 0.34 & 0.15 & 0.03 & 0.11 & 29.26 & -2.93 \\
\hline $228 \ldots \ldots$ & H II 2147 & 0.76 & $\ldots$ & 27. & 0.52 & 0.02 & 0.11 & 0.02 & 30.60 & -2.82 \\
\hline $229 \ldots \ldots$ & HCG 355 & $\ldots$ & 2.70 & $\ldots$ & 0.14 & 0.14 & 0.03 & 0.13 & 28.83 & -2.85 \\
\hline $231 \ldots \ldots$ & H II 2172 & 0.58 & 0.39 & 10. & $\cdots$ & $\ldots$ & -0.21 & 0.17 & 28.30 & -4.25 \\
\hline $234 \ldots \ldots$ & H II 2208 & 1.32 & 1.64 & 73. & 0.27 & 0.12 & 0.21 & 0.09 & 28.42 & -3.05 \\
\hline $237 \ldots \ldots$ & H II 2244 & 0.95 & 0.92 & 46. & 0.76 & 0.07 & 0.16 & 0.05 & 28.99 & -2.82 \\
\hline $241 \ldots \ldots$ & H II 2278 & 0.83 & 0.67 & $<\theta$. & $\ldots$ & $\ldots$ & 0.20 & 0.17 & 29.58 & -3.82 \\
\hline $243 \ldots \ldots$ & HCG 370 & $\cdots$ & 2.24 & $\ldots$ & $\ldots$ & $\ldots$ & 0.04 & 0.20 & 28.82 & -3.29 \\
\hline $247 \ldots \ldots$ & HCG 372 & 1.48 & 2.41 & $\ldots$ & 0.40 & 0.17 & -0.08 & 0.17 & 29.05 & -2.87 \\
\hline $249 \ldots \ldots$ & HCG 375 & $\cdots$ & 2.48 & $\cdots$ & 0.33 & 0.11 & 0.00 & 0.12 & 29.00 & -2.69 \\
\hline $260 \ldots \ldots$ & H II 2366 & 0.78 & 0.59 & $<7$. & 0.33 & 0.11 & 0.00 & 0.12 & 28.85 & -4.32 \\
\hline
\end{tabular}


TABLE 3-Continued

\begin{tabular}{|c|c|c|c|c|c|c|c|c|c|c|}
\hline $\begin{array}{c}\text { X-ray } \\
\text { Number } \\
(1)\end{array}$ & $\begin{array}{c}\text { Star } \\
\text { Name } \\
(2)\end{array}$ & $\begin{array}{c}(B-V)_{0} \\
(3)\end{array}$ & $(V-I)_{0}$ & $\begin{array}{c}v \sin i \\
\left(\mathrm{~km} \mathrm{~s}^{-1}\right) \\
(5)\end{array}$ & $\begin{array}{l}H R_{1} \\
(6)\end{array}$ & $\begin{array}{l}\sigma_{1} \\
(7)\end{array}$ & $\begin{array}{r}H_{2} R_{2} \\
(8)\end{array}$ & $\begin{array}{l}\sigma_{2} \\
(9)\end{array}$ & $\begin{array}{c}L_{X} \\
\left(\operatorname{ergs~s}{ }^{-1}\right) \\
(10)\end{array}$ & $\begin{array}{l}\frac{L_{X}}{L_{b \bullet 1}} \\
(11)\end{array}$ \\
\hline $253 \ldots \ldots$ & H II 2407 & 0.91 & 0.75 & $<7$ & $\ldots$ & $\cdots$ & 0.07 & 0.14 & 29.16 & -3.78 \\
\hline $266 \ldots \ldots$ & H II 2548 & 1.29 & 1.39 & $<9$ & $\cdots$ & $\ldots$ & 0.04 & 0.20 & 28.98 & -3.52 \\
\hline $268 . \ldots \ldots$ & H II 2602 & 1.59 & 2.25 & 11. & 0.53 & 0.16 & 0.04 & 0.15 & 29.42 & -2.87 \\
\hline $269 \ldots \ldots$ & H II 2601 & 1.49 & 2.12 & 10. & 0.42 & 0.11 & 0.03 & 0.11 & 29.37 & -3.05 \\
\hline $270 \ldots \ldots$ & H II 2588 & 1.14 & 1.13 & $<7$ & 0.44 & 0.16 & 0.21 & 0.15 & 29.20 & -3.42 \\
\hline $272 \ldots \ldots$ & HCG 394 & 1.93 & 2.74 & $\ldots$ & $\ldots$ & $\cdots$ & 0.07 & 0.23 & 28.95 & -2.74 \\
\hline $274 \ldots \ldots$ & H II 2644 & 0.73 & 0.49 & $<7$ & $\cdots$ & $\cdots$ & -0.06 & 0.18 & 29.04 & -4.27 \\
\hline $280 \ldots \ldots$ & H II 2741 & 0.97 & 0.87 & 10. & $\ldots$ & $\cdots$ & 0.02 & 0.10 & 29.38 & -3.41 \\
\hline $282 \ldots \ldots$ & H II 2786 & 0.56 & $\cdots$ & 25. & $\cdots$ & $\cdots$ & -0.12 & 0.15 & 29.38 & -4.21 \\
\hline $287 \ldots \ldots$ & H II 2881 & 0.93 & 0.84 & 12. & 0.50 & 0.09 & 0.01 & 0.08 & 29.90 & -3.30 \\
\hline $289 \ldots \ldots$ & H II 2880 & 0.82 & $\cdots$ & $<7$ & $\cdots$ & $\ldots$ & -0.20 & 0.24 & 28.98 & -4.09 \\
\hline $292 \ldots \ldots$ & H II 2927 & 1.22 & 1.37 & 95. & $\cdots$ & $\cdots$ & -0.08 & 0.16 & 29.34 & -3.10 \\
\hline $293 \ldots \ldots$ & HCG 422 & $\cdots$ & 2.37 & $\cdots$ & 0.55 & 0.14 & 0.17 & 0.12 & 29.30 & -2.78 \\
\hline $294 \ldots \ldots$ & HCG 424 & $\cdots$ & 2.31 & $\cdots$ & 0.34 & 0.18 & -0.06 & 0.18 & 29.22 & -2.85 \\
\hline $285 \ldots \ldots$ & H II 3019 & 1.17 & 1.20 & $<\theta$. & $\cdots$ & $\cdots$ & -0.03 & 0.17 & 29.28 & -3.29 \\
\hline $296 \ldots \ldots$ & HCG 428 & $\ldots$ & 2.04 & $\cdots$ & $\cdots$ & $\cdots$ & -0.11 & 0.16 & 29.43 & -2.63 \\
\hline $297 \ldots \ldots$ & H II $\mathbf{3 0 6 3}$ & 1.13 & 1.23 & 28. & 0.42 & 0.16 & 0.09 & 0.14 & 29.61 & -2.93 \\
\hline $298 \ldots \ldots$ & H II 3096 & 0.89 & 0.76 & $\cdots$ & 0.51 & 0.08 & -0.06 & 0.07 & 29.74 & -3.23 \\
\hline $300 \ldots \ldots$ & H II 3163 & 0.97 & 0.97 & 70. & 0.50 & 0.08 & -0.02 & 0.07 & 29.78 & -2.96 \\
\hline $302 \ldots \ldots$ & H II 3179 & 0.52 & $\cdots$ & $<7$ & $\cdots$ & $\cdots$ & -0.17 & 0.18 & 29.27 & -4.42 \\
\hline $304 \ldots \ldots$ & H II 3197 & 1.03 & 1.07 & 33. & 0.54 & 0.05 & 0.03 & 0.05 & 30.14 & -2.91 \\
\hline
\end{tabular}

increasing $L_{\mathbf{X}} / L_{\text {bol }}$ for the F- and G-type stars. More significantly, the rapidly rotating $G$ stars all like in a quadrant defined by $\mathrm{HR}_{2}>0.0$ and $L_{\mathrm{x}} / L_{\text {bol }}>-3.3$, while the $F$ stars and slowly rotating $G$ stars generally lie in the opposite quadrant. If we interpret $L_{\mathrm{X}} / L_{\mathrm{bol}}$ as the efficiency of the coronal heating mechanism, then Figure $5 a$ suggests increased coronal temperatures with increased coronal heating efficiency. On the other hand, there is little we can say about the K- and M-type hardness ratios because the relative errors are so large and because we are only sampling the most active lower mass stars.

A formal analysis of the error-weighted correlations (see Bevington \& Robinson 1992) indicates that $\mathrm{HR}_{2}$ is correlated with $L_{\mathrm{x}}$ and $L_{\mathrm{x}} / L_{\mathrm{bol}}$ at the $99 \%$ confidence level for the F- and G-type Pleiades stars. For the $K$ and $M$ stars, $H_{R}$ is correlated with $L_{X}$ at the $99 \%$ confidence level. These stars have hardness ratios and activity levels more comparable to those of the rapidly rotating $\mathrm{G}$ stars.

In conclusion, the analysis of the individual $\mathrm{X}$-ray spectra, the composite X-ray spectra, and the hardness ratios derived for the late-type stars all point to cooler coronal temperatures and lower activity levels for $F$ stars and slowly rotating G-type dwarfs when compared to those of the K-, M-, and more rapidly rotating G-type dwarfs.

As has been noted, these results are similar to those found by Schmitt et al. (1990) for late-type field stars. When comparing overall X-ray activity from field stars and open clusters, $L_{\mathbf{X}}$ has been shown, overall, to decrease with age (e.g., Rosner, Golub, \& Vaiana 1985). The similarity, then, between the coronal distributions in the Pleiades and the stars examined by Schmitt et al. (1990) may appear somewhat surprising. We point out,

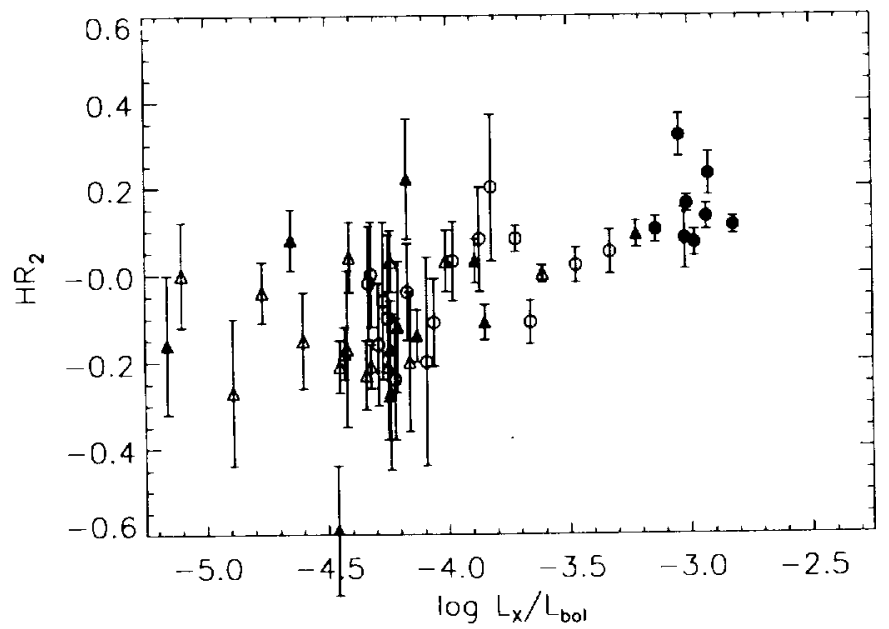

FIG. $5 a$

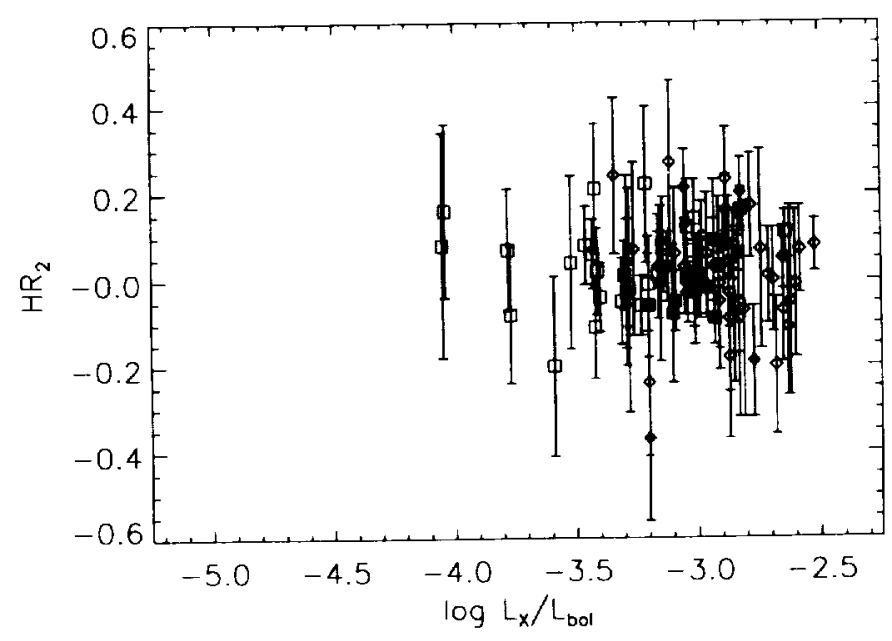

Fig. $5 b$

Fig. 5. $-\mathrm{HR}_{2}$ vs. $L_{\mathrm{x}} / L_{\mathrm{bol}}$ for $(a) F$ stars and $G$ stars and $(b) K$ stars and $M$ stars. See Fig. 4 for symbol definitions. 
however, that the Schmitt et al. sample is biased toward extremely bright $X$-ray sources, which, on average, have $X$-ray activity levels more comparable to those of young dwarfs like the Pleiades than of typically less active field dwarfs.

\section{X-RAY FLARES}

The ROSAT PSPC data contain the time of arrival of every detected X-ray photon. From the photon event data we have constructed background-subtracted light curves of every detected source in every one of the long exposures in Table 1 of Paper I, allowing us to look for source variability on timescales as short as $500 \mathrm{~s}$.

Each light curve was visually inspected for signs of variability or obvious flaring. We also used a simple $\chi^{2}$ test on each light curve to test the null hypothesis of a constant count rate. While a number of sources were found not to be constant (above the $99 \%$ confidence level), only half or so of these showed the characteristic rise and decay of a strong stellar flare. We found no sources showing strong evidence of periodic variability.

In Figure 6 we present light curves for the 12 most convincing cases of X-ray flaring in the PSPC data. In Figure 6 we plot the observed count rate (not corrected for telescope vignetting) with $1 \sigma$ error bars against time from the beginning of the observation. Ten of these flares are associated with known, late-type Pleiades cluster members. The most intriguing of the flares is associated with source 191. This source has no cataloged stellar counterpart; however, a very faint star $(V>16)$ appears less than $10^{\prime \prime}$ away on the Palomar Observatory Sky Survey E plate of the region (see Fig. 8 of Paper I). If the counterpart to the observed flare is indeed a Pleiades cluster member, then the peak X-ray luminosity during flare would have reached $\sim 10^{31}$ ergs $^{-1}$.

All of the flares associated with Pleiades cluster members had peak flare luminosities in excess of $10^{30}$ ergs $s^{-1}$, the brightest one, on the G star H II 2147 , with $L_{\mathrm{X}}^{\text {eeak }} \sim 2.5 \times 10^{31}$ ergs $\mathrm{s}^{-1}$. The flares are generally characterized by rise times in the range $(1-10) \times 10^{3} \mathrm{~s}$, decay times in the range $(5-15) \times 10^{3}$
S, and decay times typically exceeding rise times by a factor of $\sim 3$.

In Table 4 we summarize our findings for the dozen flares in Figure 6. In columns (1)-(4) we list the X-ray number, star name, $(B-V)_{0}$, and $(V-I)_{0}$ colors of the optical counterpart. In columns (5)-(8) we list the quiescent and peak $X$-ray luminosities as well as the $e$-folding times of the rise and decay phases of the flare. Only two flare spectra contained sufficient counts to model the peak of the emission: H II 2147 and H II 1516 (see $\S 3.1$ below).

For the sake of consistency, we have assumed the same count rate to luminosity conversion factor as was used in Paper I for all the flares in Table 4. Since stellar flares are nearly always characterized by temperatures in excess of $1 \mathrm{keV}$ (i.e., $\gtrsim 12 \times 10^{6}$ K) (Poletto, Pallavicini, \& Kopp 1988; van den Oord \& Mewe 1989; Fisher \& Hawley 1990; Schmitt 1994), we have probably underestimated the true count-rate to luminosity conversion factor. Consequently, the $L l^{\text {peak }}$ values in Table 4 are lower limits. The rise-time and decay-time upper limits in Table 4 indicate that the rise or the decay phase of the flare was not sufficiently monitored to determine an $e$-folding time.

Overall, the Pleiades flares are among the brightest $\mathrm{X}$-ray flares ever reported on single, late-type main-sequence dwarfs. Stellar X-ray flares with $L_{\mathbf{X}} \sim 10^{31}$ ergs $\mathrm{s}^{-1}$ have only been reported for the eclipsing binary $\beta$ Per (Algol) (van den Oord \& Mewe 1989), for the flare star AU Mic (Cully et al. 1994), and for a handful of pre-main-sequence stars in $\rho$ Oph (Montmerle et al. 1983) and Orion (Gagné et al. 1995) and for the Pleiades star H II 1136 as observed by the Einstein IPC (Caillault \& Helfand 1985). During the ROSAT PSPC pointed observation, H II 1136 was not variable above the $99 \%$ confidence limit, and no flare was apparent.

Comprehensive surveys of Einstein and EXOSAT observatious of stellar X-ray flares have been presented by Haisch (1983) and Pallavicini, Tagliaferri, \& Stella (1990), respectively. While most of those X-ray flares occurred on dMe stars, the $R O S A T$ all-sky survey has found that large flares occur on all types of late-type stars (Schmitt 1994). Our results confirm the

TABLE 4

X-RAY Flare Stars

\begin{tabular}{|c|c|c|c|c|c|c|c|}
\hline \multirow{2}{*}{$\begin{array}{c}\text { X-Ray } \\
\text { Number } \\
(1)\end{array}$} & \multirow[b]{2}{*}{$\begin{array}{c}\text { Star } \\
\text { Name } \\
(2)\end{array}$} & \multirow[b]{2}{*}{$\begin{array}{c}(B-V)_{0} \\
(3)\end{array}$} & \multirow[b]{2}{*}{$\begin{array}{c}(V-I)_{0} \\
(4)\end{array}$} & \multicolumn{2}{|c|}{$\begin{array}{c}\log L_{x} \\
\left(\operatorname{ergs} s^{-1}\right)\end{array}$} & \multicolumn{2}{|c|}{$\begin{array}{c}\text { Duration } \\
\left(10^{3} \mathrm{~s}\right)\end{array}$} \\
\hline & & & & $\begin{array}{l}\text { Quiet } \\
\text { (5) }\end{array}$ & $\begin{array}{c}\text { Peak } \\
\text { (6) }\end{array}$ & $\begin{array}{c}\text { Rise } \\
(7)\end{array}$ & $\begin{array}{c}\text { Decay } \\
(8)\end{array}$ \\
\hline $9 . \ldots$ & HCG 97 & $\cdots$ & 2.02 & 29.2 & 30.2 & $<3.4$ & 5.0 \\
\hline $32 \ldots \ldots$ & H II 174 & 0.81 & $\cdots$ & $\mathbf{3 0 . 0}$ & 30.5 & 5.5 & 13.5 \\
\hline 35..... & H II 191 & 1.31 & 1.61 & 29.3 & 30.5 & 1.0 & 5.0 \\
\hline 36..... & H II 212 & 1.33 & 1.51 & 28.3 & 30.3 & $<4.0$ & 6.0 \\
\hline $56 \ldots$. & HCG 143 & 1.61 & 2.37 & 29.6 & $30.4^{2}$ & 0.5 & 1.5 \\
\hline $62 \ldots \ldots$ & H II 345 & 0.80 & $\cdots$ & 30.1 & 30.7 & $<4.0$ & 6.5 \\
\hline $90 \ldots \ldots$ & HCG 181 & 1.53 & 2.28 & 29.5 & 30.5 & 1.0 & 5.0 \\
\hline $142 \ldots \ldots$ & H II 1100 & 1.06 & 1.02 & 29.7 & $30.5^{b}$ & 3.0 & 9.5 \\
\hline $187 \ldots \ldots$ & H II 1516 & 1.30 & 1.43 & 29.9 & 31.2 & $<5.0$ & 5.0 \\
\hline $181 \ldots \ldots$ & $\cdots$ & $\cdots$ & $\cdots$ & 29.7 & $30.9^{c}$ & $<4.5$ & 11.0 \\
\hline $228 \ldots \ldots$ & H II 2147 & 0.76 & $\cdots$ & 30.6 & $31.4^{b}$ & $<11.0$ & $<84.0$ \\
\hline $237 \ldots \ldots$ & H II 2244 & 0.95 & 0.92 & 29.9 & 30.6 & $<5.0$ & 8.0 \\
\hline
\end{tabular}

- Although the membership of HCG 143 is uncertain, we assume a distance of $127 \mathrm{pc}$

- The peak of the flare was probably not observed.

c Although this source has no optical counterpart, we assume a distance of $127 \mathrm{pc}$. 


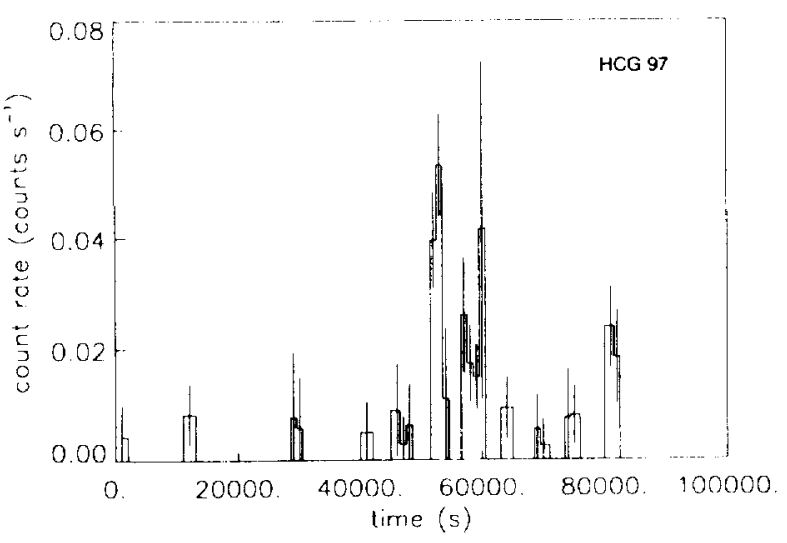

FIG. 6a

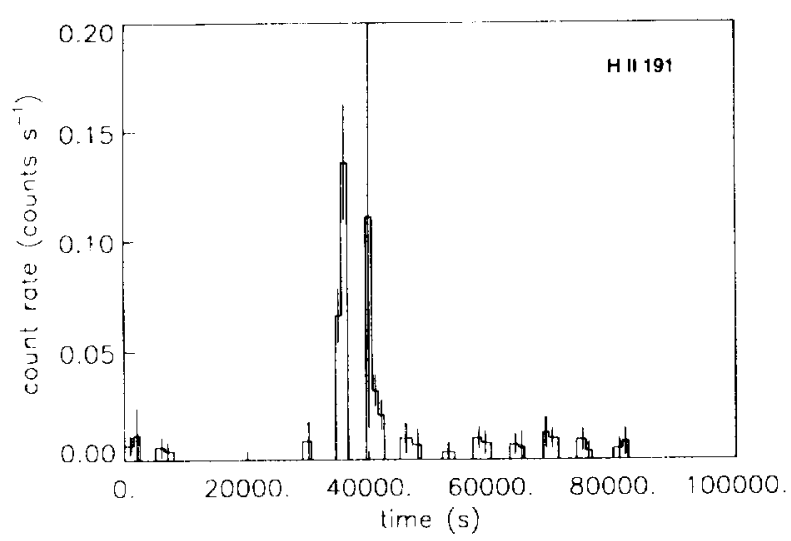

Fig. $6 c$

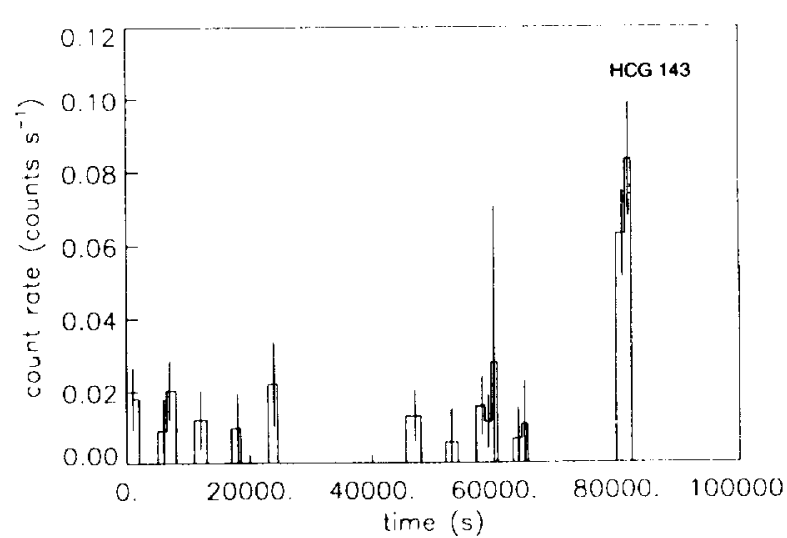

Fig. $6 e$

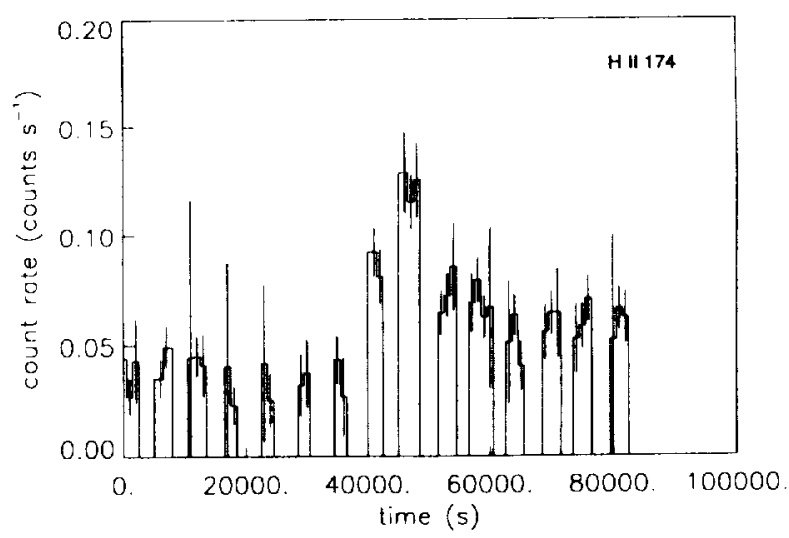

Fig. $6 b$

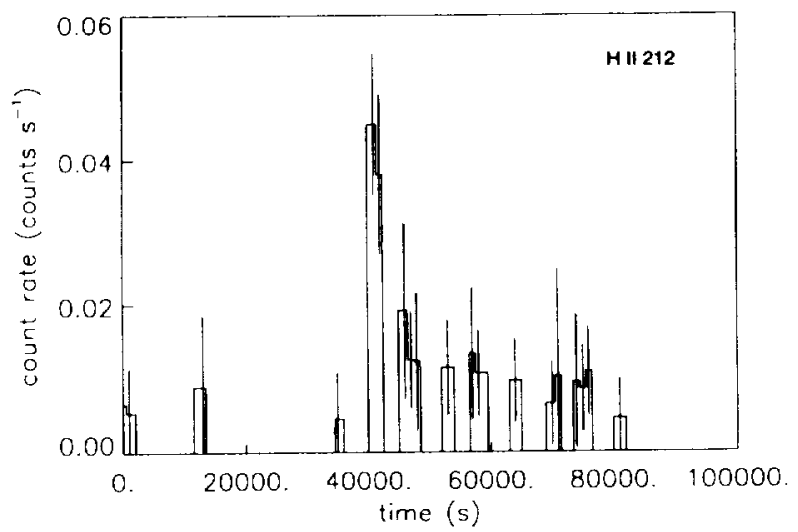

Fig. $6 d$

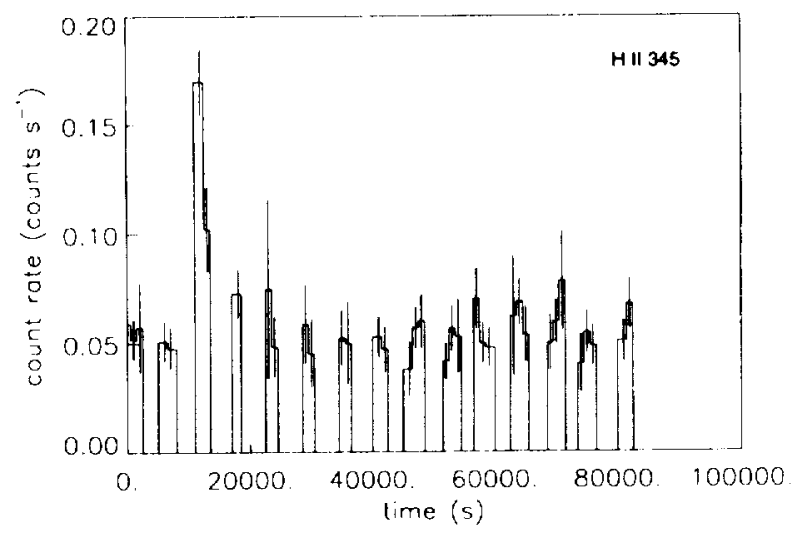

Fic. $6 f$

Fig. 6.-Light curves for the dozen strong X-ray flares detected in the ROSAT PSPC data of the Pleiades. Plotted are the uncorrected PSPC count rates vs. time

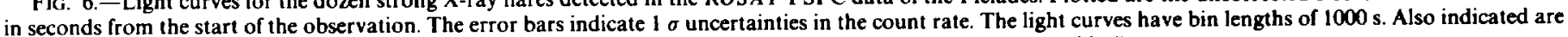
the field from which the light curve was extracted, the observation start date, and the X-ray source number (see Table 4).

all-sky survey finding with flares detected on dwarfs ranging in spectral type from $\sim$ G8 (H II 2147) to $\sim$ M3 (HCG 143) and ranging in rotational velocity from $v \sin i<9 \mathrm{~km} \mathrm{~s}^{-1}$ (H II 1100) to $\sim 45 \mathrm{~km} \mathrm{~s}^{-1}$ (H II 2244).

Although our sample of detected $\mathrm{X}$-ray flares is small and strongly biased toward very powerful events, we can begin to estimate the flaring frequency of the Pleiades dwarfs. The large flares we detect had typical durations (rise + decay) of $10^{4} \mathrm{~s}$ with typical monitoring periods of $\sim 6 \times 10^{4} \mathrm{~s}$. Of the 171 detected cluster members, 10 were seen to flare. We conclude that the flaring frequency for large $\left(L_{x} \gtrsim 10^{30} \mathrm{ergs} \mathrm{s}^{-1}\right)$, longduration $\left(t \sim 10^{4}\right.$ s) flares is about $1 \%$. This appears to be higher than the flaring frequency observed in the Hyades (Stern et al. 1994) and may be indicative of the comparatively lower activity levels of the Hyades dwarfs. This decreased $\mathrm{X}$-ray activity is presumably the result of the overall decrease 


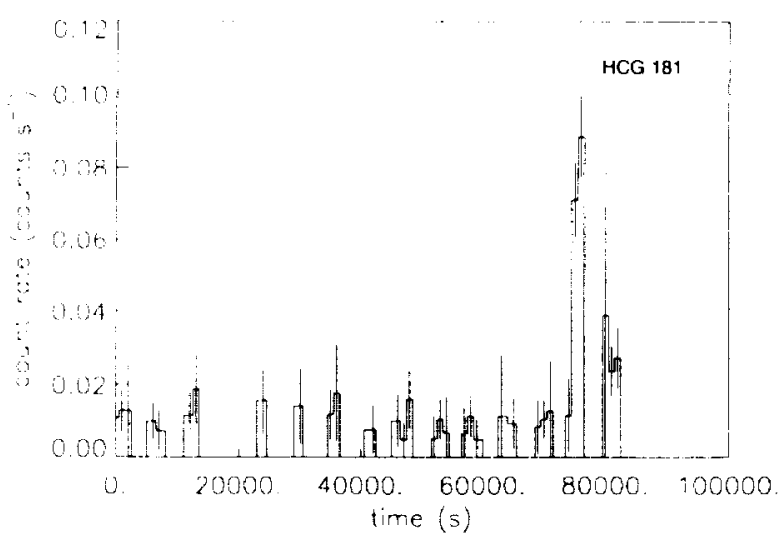

FIG. 69

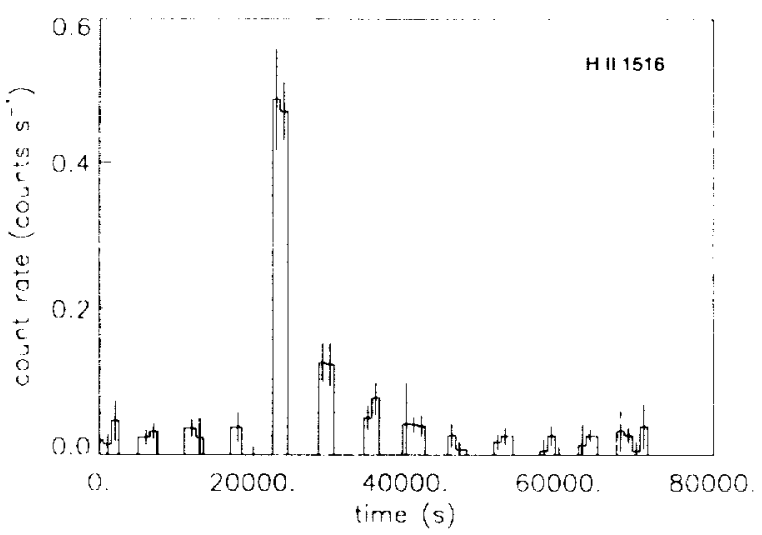

FiG. $6 i$

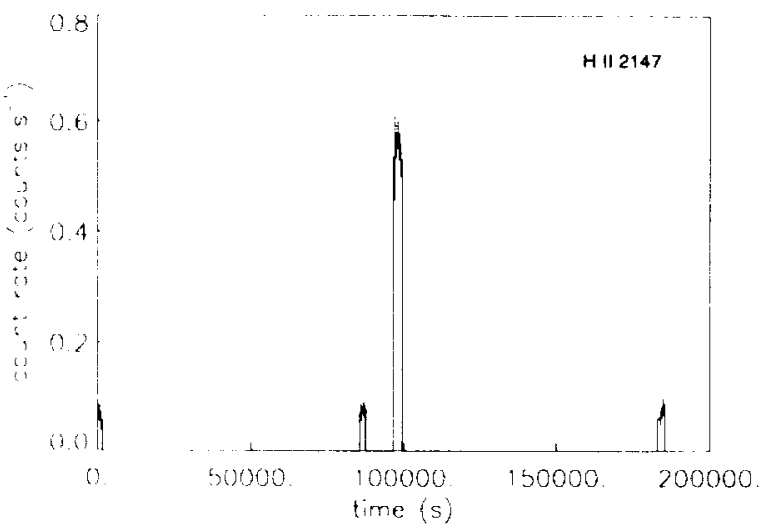

Fig. $6 k$

in rotation rates from the age of the Pleiades $\left(t_{*} \sim 70 \mathrm{Myr}\right)$ to the age of the Hyades $\left(t_{*} \sim 600 \mathrm{Myr}\right)$.

\subsection{Modeling the Flares on H II 2147 and H II 1516}

Two of the detected flares had sufficiently high count rates to model the PSPC spectrum of the flare peak. In the case of $\mathbf{H}$ II 2147 , the flare was only seen during one $R O S A T$ orbit with a total exposure time of $\sim 1900 \mathrm{~s}$. The $\sim 1000$ net counts in the flare spectrum were modeled with a two-temperature RS model, the results of which we present in Table 1 . Since the rise and decay of the flare were not observed, there is little else we

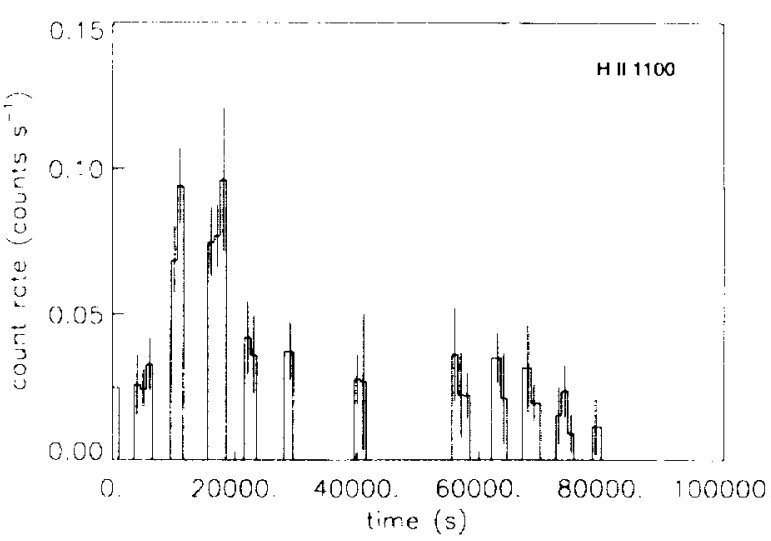

FIG. $6 h$

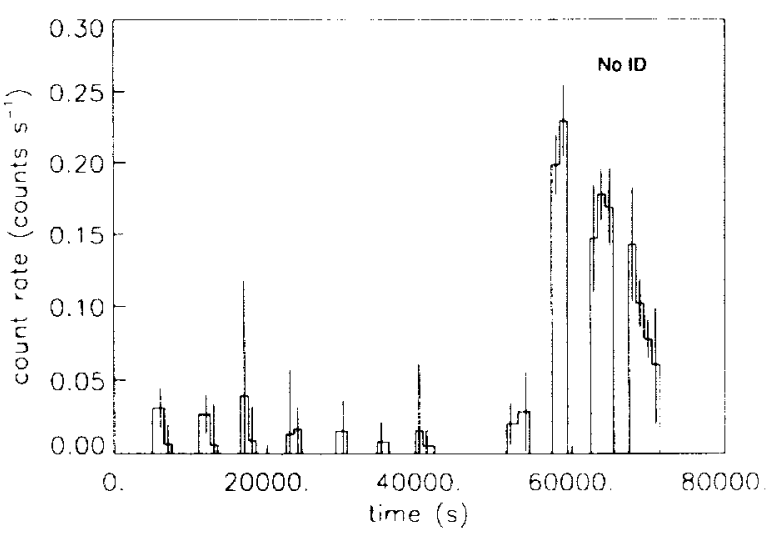

FIG. $6 j$

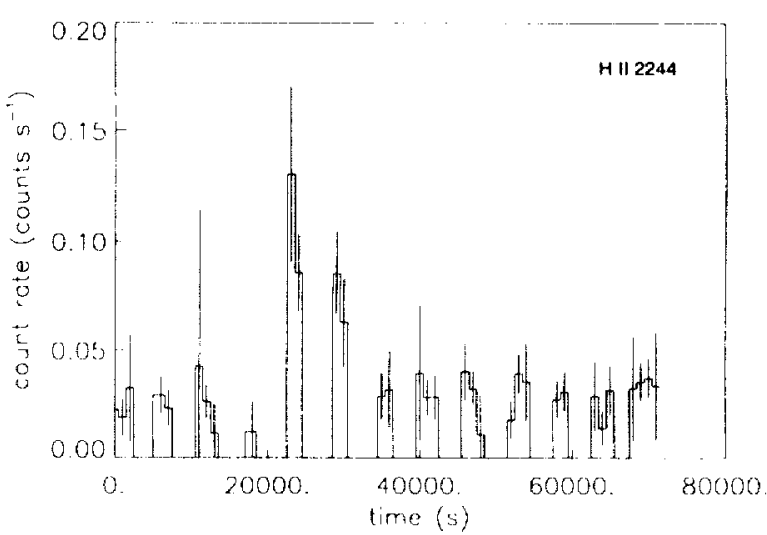

FIG. 61

can infer from the H II 2147 light curve. We point out the enormous emission measure, $E M \gtrsim 2 \times 10^{54} \mathrm{~cm}^{-3}$, during flare peak.

The second brightest flare is associated with the late-K type Pleiades star H II 1516. We estimate the e-folding time of the decay phase to be $\sim 5.0 \times 10^{3} \mathrm{~s}$. The peak of the flare was observed for $\sim 660 \mathrm{~s}$ during one $\operatorname{ROSAT}$ orbit. The $\sim 400$ net counts during this orbit were modeled with a one-temperature RS model. Although the signal-to-noise ratio of the PSPC spectrum of the flare peak is very low, the minimum flare temperature and emission measure we obtain (at the $68 \%$ con- 
fidence level) are $k T \gtrsim 1.1 \mathrm{keV}$ and $1.2 \times 10^{54} \mathrm{~cm}^{-3} \lesssim \mathrm{EM} \lesssim$ $2.4 \times 10^{54} \mathrm{~cm}^{-3}$. The flare temperature $(T \gtrsim 13 \mathrm{MK})$ is typical of large flares on late-type dwarfs. We cannot constrain the temperature of a very hot plasma because of the low signal-tonoise ratio of the X-ray spectrum and the low sensitivity of the PSPC at energies above $\sim 1.4 \mathrm{keV}$. As a result, we estimate the peak temperature to be $T_{0} \sim 25 \mathrm{MK}$, a typical value for latetype flares (Schmitt 1994; Pallavicini et al. 1990). As was the case for H II 2147, the volume emission measure of H II 1516 at the onset of the flare, $E M \sim 2 \times 10^{54} \mathrm{~cm}^{-3}$, is very large.

We can use the observed $e$-folding time of the decay phase to estimate the average electron density and the total volume of the plasma at the onset of the flare. In the quasi-static cooling loop model (van den Oord \& Mewe 1989), one assumes that the flaring plasma decays through a series of stationary loops at constant volume and pressure. These assumptions are in contrast to the modelling schemes of Poletto et al. (1988) and Fisher \& Hawley (1990). However, since the latter two models are more complex and, hence, require more detailed knowledge of the flare decay, we will limit our analysis to quasi-static cooling.

We follow Schmitt (1994) and define two characteristic timescales for the decay phase: the observed $e$-folding time, $\tau_{d}$, and the radiative cooling time defined as

$$
\tau_{c}=\frac{3 k T_{0}}{n_{0} \Lambda\left(T_{0}\right)} .
$$

where $T_{0}, n_{0}$, and $\Lambda\left(T_{0}\right)$ are the initial plasma temperature, mean electron density, and radiative cooling law, respectively, at the beginning of the decay phase. In the quasi-static loop model, if one assumes no additional heating during the decay phase, the time evolution of the total radiative output reduces to a particularly simple form:

$$
E_{\mathrm{rad}}(t)=E_{0}\left(1+\frac{t}{3 \tau_{c}}\right)^{-4}
$$

At $t=\tau_{d}, E_{\text {rad }}\left(\tau_{d}\right) / E_{0}=e^{-1}$. Consequently, we can infer the radiative cooling time directly from the decay time,

$$
\tau_{c}=\frac{\tau_{d}}{3\left(e(1 / 4)^{1 / 2}-1\right)} \simeq 1.2 \tau_{d}
$$

In the case of $H$ II $1516, \tau_{d} \sim 5.0 \times 10^{3} \mathrm{~s}$. Using an estimated temperature $T_{0} \sim 2.5 \times 10^{7} \mathrm{~K}$, assuming a cooling law appropriate for this temperature regime $\Lambda\left(T_{0}\right) \sim 1.3 \times 10^{-23}$ ergs $\mathrm{cm}^{3} \mathrm{~s}^{-1}$ (van den Oord \& Mewe 1989), substituting for $\tau_{d}$ in equation (6), and solving for equation (4), we obtain a mean electron density $n_{0} \sim 1.3 \times 10^{11} \mathrm{~cm}^{-3}$. This is certainly a lower limit to the peak electron density because the true peak plasma temperature may, in fact, be higher than our estimate.

If we assume a constant electron density throughout the flare loop, we infer from the peak observed emission measure, $\mathrm{EM} \sim 2 \times 10^{54} \mathrm{~cm}^{-3}$, a total plasma volume $V \sim 10^{32} \mathrm{~cm}^{3}$. If the flare is the result of a single coronal loop with aspect ratio (ratio of loop diameter to loop length) $\alpha \sim 0.1$ (see van den Oord \& Mewe 1989), then the inferred loop size is huge, $L \sim 3 \times 10^{11} \mathrm{~cm}$. That is, $L \geqslant 6 R_{*}$. Of course, the flare may be the result of multiple loops, in which case the loop length can be scaled down accordingly.

We point out, though, that the large plasma volume may be the result of our underestimate of the peak flare temperature or may be an artifact of the quasi-static cooling loop model. As has been pointed out by Schmitt (1994), alternative models like the two-ribbon flare model of Poletto et al. (1988) lead to higher electron densities and more compact loop structures. Higher energy X-ray spectra and more complete temporal coverage of flare events are required to model $X$-ray flares correctly.

\section{MEDIUM- AND LONG-TERM X-RAY VARIABILITY}

The PSPC data for the Pleiades were collected in exposures separated by $6-18$ months. The Pleiades was also monitored by Einstein more than a decade ago, allowing us to look at a particular source's variability on medium- and long-term timescales.

Throughout this section we employ a procedure for estimating the cumulative count-rate ratio distribution given a pair of observations of a set of X-ray sources first used by Schmitt et al. (1993) and subsequently by Gagné \& Caillault (1994) and Gagné et al. (1995).

For each pair of observations of a particular source, we compute a count-rate ratio, $r \equiv \eta_{1} / \eta_{2}$, where $\eta_{1}$ and $\eta_{2}$ are the count rates or upper limits from the earlier and later observation, respectively. Since some sources detected in one exposure are observed but not detected in others, the count-rate ratios consist of some upper and lower limits.

We bin the three sets of $\log r$ values into three distributions (count-rate ratios, upper limits, and lower limits). The set of $r$-values for all observations are doubly censored univariate data, and we estimate the "true" distribution of count-rate ratios iteratively using the maximum likelihood estimator described by Schmitt et al. (1993)

\subsection{Medium-Term Variability: I Year Timescale}

The PSPC exposures of the northeast and northwest fields were all observed in 1991 September. However, the observations of the center field were done in three segments: 1991 February, 1991 August, and 1992 August with exposure times of $\sim 4, \sim 16$, and $\sim 7 \mathrm{ks}$, respectively. This has allowed us to look at count-rate variations on three timescales: 6 months between 1991 February and 1991 August, 12 months between 1991 August and 1992 August, and 18 months between 1991 February and 1992 August. To eliminate any bias due to the different exposure times of the three observations, we have further divided the individual exposures into appropriately long blocks. That is, to compare the 1991 February and 1991 August data sets, the 1991 August data have been divided into four contiguous blocks of $\sim 4 \mathrm{ks}$.

For each $\mathrm{X}$-ray source associated with a single Pleiades cluster member, we have determined a count-rate or $3 \sigma$ upper limit in each block of time. For each pair of observations, a count-rate ratio has been determined. Because of the presence of upper limits in both the "before" and "after" data, the count-rate ratios consist of ratios, upper limits, and lower limits. Ratios determined from two upper limits have been discarded. We have used the iterative maximum likelihood technique described by Schmitt et al. (1993) and Gagné \& Caillault (1994) to estimate the true count-rate ratio distribution.

In all cases, the distributions are symmetric about $\log r \sim 0$ and, hence, have been folded about $\log r=0$ to construct cumulative distributions. In Figure 7 we present the resulting cumulative count-rate ratio distributions from observations separated by 18 months (top panel), 12 months (middle panel), and 6 months (bottom panel) as solid histograms. 

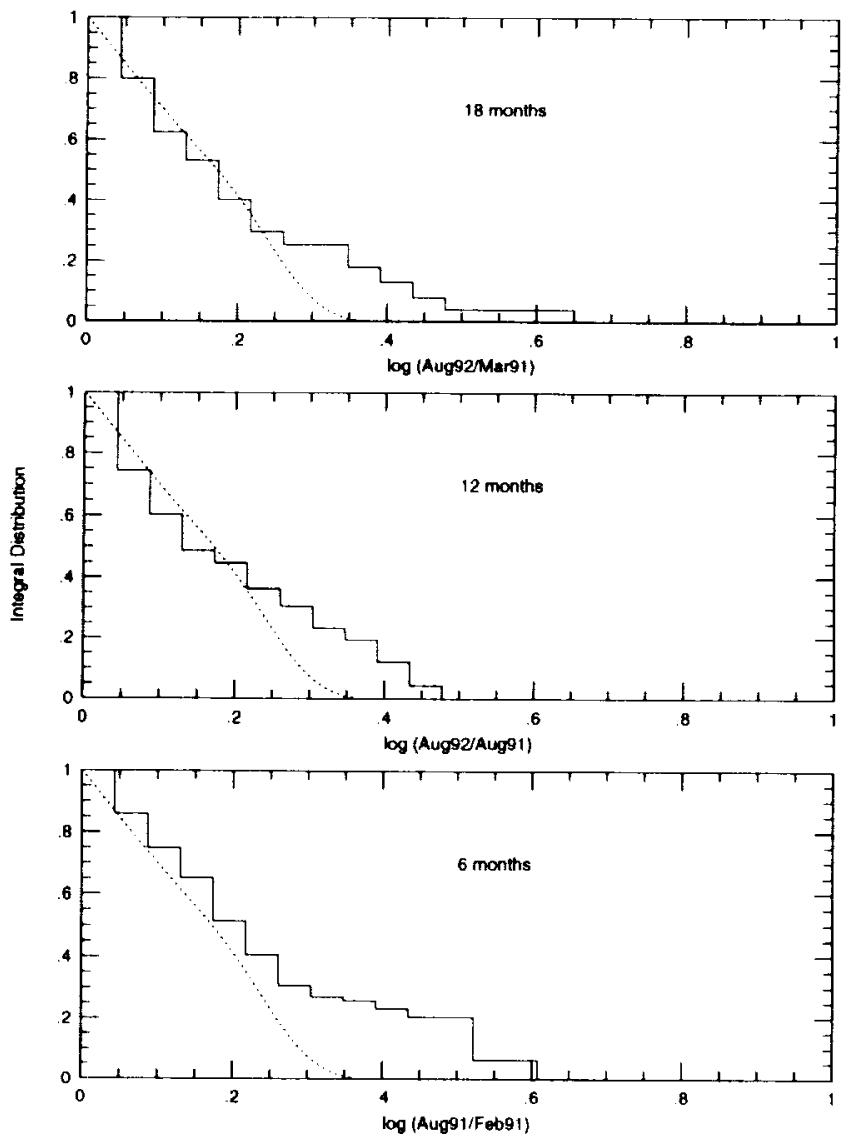

FIG. 7. Count-rate ratio histograms for sources associaled with late-type Pleiades stars from observations separated by 18 months (top pane), 12 months (middle panel), and 6 months (bottom panel). The solid histograms represent the cumulative maximum likelihood count-rate ratio distribution for detections and upper limits using the estimator for doubly biased univariate data described by Schmitl et al. (1993). The dotted lines indicate the cumulative count-rate distribution we expect as the result of statistical fluctuations alone in the observed data in the case of a faint source with an intrinsic mean count rate at the $3 \sigma$ detection threshold.

If the $\mathrm{X}$-ray count rates were very precise, then the resulting distribution of count-rate ratios would indicate intrinsic X-ray variability. However, since many of the sources are detected at or near the source detection threshold, repeated measurements of an intrinsically constant source will produce a broad distribution of count-rate ratios as the result of measurement errors alone. We want to estimate how much of the observed variability is intrinsic and how much is the result of statistical fluctuations alone. Although the fluctuations from a photon counter like the PSPC are, strictly speaking, Poisson distributed, all the sources in this survey have been detected with at least 20 net photons; thus, we assume that a Poisson error distribution can be accurately approximated by an appropriate Gaussian distribution.

We consider the case of a source with a constant intrinsic count rate near the $3 \sigma$ source detection threshold, where $\sigma$ is the total uncertainty in the source and background counts (see Gagné \& Caillault 1994). In this case, the probability of a measurement yielding an upper limit is 0.5 , and, after repeated measurements, variations due to statistical fluctuations alone will result in count-rate ratios as well as some upper and lower limits. The resulting distribution of count-rate ratios, upper limits, and lower limits is used to calculate a maximum likelihood distribution as before.

In Figures 7, 8, and 9 (see below) we indicate the estimated cumulative distribution due to "errors only" with dotted lines. One of the important consequences of such an error analysis is that, because of the low signal-to-noise ratio of the detected sources, count-rate variations of less than a factor of 2 may not be the result of intrinsic variability but, rather, may be the result of measurement uncertainties.

In Figure 7 the difference between the solid histograms and dotted curves represents a lower limit to the true variability of the X-ray sources. As an ensemble, $Z \frac{1}{4}$ of the Pleiades stars are variable by more than a factor of 2 on all three timescales. Since the three distributions are so similar, we have combined the data to increase our sample size.

In Figure 8 we present the combined cumulative count-rate ratio histogram indicating the observed variability on 6-18 month timescales. The error bar is a typical $1 \sigma$ uncertainty in each $\log r$ bin. Uncertainties in the cumulative distribution were determined by adding and subtracting $1 \sigma$ uncertainties in the individual count rates, redetermining a set of count-rate ratios (with upper and lower limits) and recomputing the cumulative maximum likelihood distribution in each case.

Since only two strong flares (H II 2147 and H II 1100) were identified in the center field, the variability observed in over one-quarter of the observations is not only the result of very strong $X$-ray flaring. Since we have not identified more moderate flaring $\left(L_{\mathrm{x}} \leqslant 10^{30} \mathrm{ergs} \mathrm{s}^{-1}\right)$, we can only guess at the origin of this variability. We can eliminate fluctuations in the PSPC detector sensitivity as a possible explanation because such variations would have affected all the count rates determined at a particular time. This, in turn, would have skewed the $\log r$ values, giving rise to an asymmetric count-rate ratio distribution. This was not observed. We can also eliminate any uncertainties in the off-axis response of the PSPC because all the count rates were determined from the center field alone. That is, count rates for a given source were all collected from the same position on the detector using identical source and background cells.

Our finding that about one-fourth of the late-type Pleiades stars vary by more than a factor of 2 on timescales $\sim 1$ yr is in

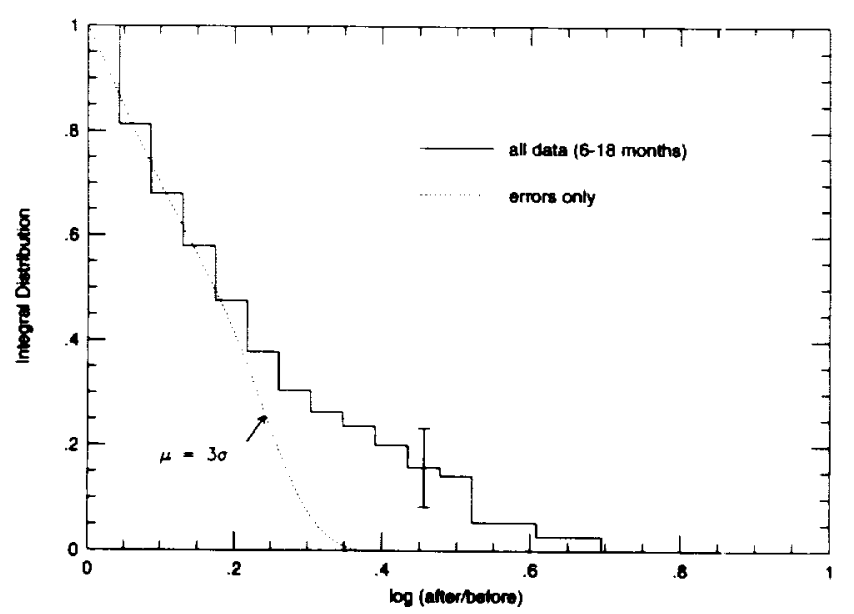

FIG. 8.-Combined cumulative count-rate ratio histogram for sources associated with late-type cluster members. Overall, one-fourth or more of the Pleiades stars are significantly variable (by more than a factor of 2) on 6-18 month timescales 
contrast to the results of Micela et al. $(1990,1995)$, who found little significant variability in their analyses of the entire Einstein data set and a single ROSAT PSPC pointing of the southwest portion of the cluster.

On the other hand, the fraction of variable Pleiades dwarfs presented herein is very similar to that observed among the very active pre-main-sequence stars in the Orion Nebula region by Gagné \& Caillault (1994) and Gagné et al. (1995). In a qualitative sense, the variability in the Pleiades is also reminiscent of the X-ray luminosity variations seen in the $\rho$ Oph star-forming region (Montmerle et al. 1983), albeit on a smaller scale.

\subsection{Long-Term Variability: 11 Year Timescale}

The Pleiades open cluster was the object of intense monitoring by the Einstein IPC, the most comprehensive results of which have been reported by Micela et al. (1990). In their analysis of the ROSAT all-sky survey data of the Pleiades, Schmitt et al. (1993) found that $\sim 40 \%$ of the sources were variable by more than a factor of 2 in the $11 \mathrm{yr}$ time span between the all-sky and Einstein observations, and suggested that the variability and the decade-long time span between observations may be indicative of solar-like activity cycles. Solar-like activity cycles have been observed in some late-type dwarfs from monitoring of chromspheric $\mathrm{Ca}$ II and $\mathrm{Mg}$ II flux variations and from optical continuum variations due to spot activity (Wilson 1978; Dorren \& Guinan 1994; Baliunas et al. 1995).

Although the Einstein observations of the Pleiades had varying exposure times, $\mathrm{X}$-ray luminosities were typically $L_{\mathrm{x}} \gtrsim 1.5 \times 10^{29} \mathrm{ergs}^{-1}$, a factor of 2 or 3 less sensitive than the $R O S A T$ pointed observations. In order to avoid possibly biasing our analysis, we consider only cluster members that could have been detected in both sets of observations of the detected sources reported by Micela et al. (1990), 69 were observed in one of the three pointed ROSAT observations.

We thus consider the 69 brightest Pleiades stars detected by ROSAT and observed by Einstein. Of these, 51 were detected by Einstein and 18 had upper limits. Of the remaining 18 Einstein detections, 14 were detected by ROSAT and four yielded only ROSAT upper limits (see Table 4 of Micela et al. 1990 and Tables 7 and 8 of Paper I). We thus have 87 $(51+18+14+4=87)$ pairs of observations consisting of 65 Pleiades cluster members detected in both surveys, four members detected only by Einstein, and 18 members detected only by $R O S A T$. The resulting $\mathrm{X}$-ray luminosity ratios consist of ratios, upper limits, and lower limits.

Although the PSPC and the IPC have slightly different bandpasses, the median value of $\log L_{\mathrm{X}}^{\text {Einstein }} / L_{\mathrm{X}}^{R O S A T}$ is approximately 0.0 , indicating overall consistency in the soft $X$-ray luminosity determinations in the two surveys. We have calculated the cumulative, maximum likelihood distribution as before.

In Figure 9 we present the resulting cumulative $L_{\mathbf{x}}$ ratio distribution (solid line) and the distribution we estimate due to measurement uncertainties alone (dotted line). The error bar is a typical $1 \sigma$ uncertainty in the ratio distribution itself. Whereas $\sim 25 \%$ of the Pleiades stars are variable by more than a factor of 2 on 6-18 month timescales, up to $40 \%$ are variable on the $\sim 11$ yr timescale between Einstein and ROSAT. Similar variability was observed by Schmitt et al. (1993) in their analysis of the $\operatorname{ROSAT}$ all-sky data.

We note that the sample used to compare the Einstein and

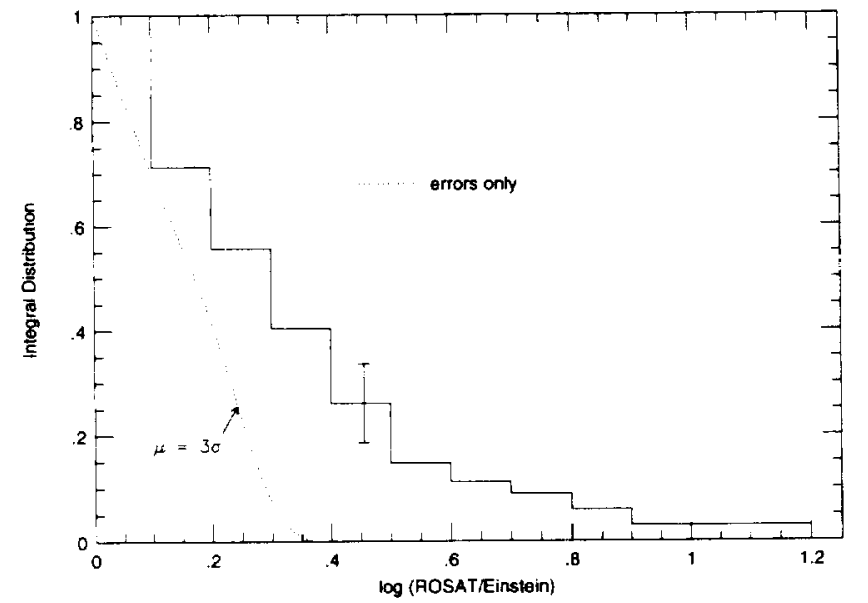

FIG. 9. Count-rate histogram for the 69 brightest sources observed by both the ROSAT PSPC and the Einstein IPC. Approximately $40 \%$ of the Pleiades stars are significantly variable (by more than a factor of 2 ) on the $\sim 11$ yr timescale between missions.

ROSAT pointed observations are brighter in $\mathrm{X}$-rays than the sample used to examine approximately $1 \mathrm{yr}$ variability in the ROSAT data alone. We have examined the effect of this bias by recomputing count-rate distributions (as was done in $\$ 4.1$ ) for a reduced sample of ROSAT sources with the same flux limit as the Einstein observations, i.e., corresponding to $L_{\mathbf{x}} \gtrsim 1.5$ $\times 10^{29}$ ergs s$^{-1}$. We find, as before, that approximately $25 \%$ of sources are variable by more than a factor of 2 on roughly $1 \mathrm{yr}$ timescales. We conclude that we are seeing marginal evidence for increased activity over the decade-long timescale.

The fraction of X-ray sources in the Pleiades that do show some variability (around $25 \%$ ) do so on all timescales longer than a month. Hence, any solar-like activity-cycle signatures would manifest themselves as additional variability. The apparent increase in variability on the longer timescale may be indicative of a physical process like solar activity cycles. However, the difference in the two distributions is only marginal and, given the limited number and quality of the measured $L_{\mathrm{x}}$ values, these results, along with those of Schmitt et al. (1993), only hint at the possibility of long-term activity cycles for the late-type stars in the Pleiades.

\section{SUMMARY}

Summarizing, we have used three deep ROSAT PSPC pointed observations to examine the $X$-ray spectral and temporal characteristics of the late-type dwarfs in the Pleiades. We have modeled the PSPC pulse-height spectra of individual Xray-bright Pleiades stars with one- and two-temperature RS optically thin plasma models. The fits suggest moderately hotter coronal temperatures for $\mathbf{K}$ and rapidly rotating $\mathbf{G}$ stars than for $F$ and slowly rotating $G$ stars. For $F$-, $G$-, and $K$-type Pleiades stars, we find a general increase in coronal temperature with $L_{\mathbf{x}} / L_{\text {bol }}$. No M-type Pleiades stars were detected with sufficient counts to constrain the spectral models. Our findings are in general agreement with those of Schmitt et al. (1990) from their analysis of active late-type stars detected by the Einstein Observatory. Because the Pleiades contain a handful of very rapidly rotating $G$ stars, we have been able to show that these $G$ stars have coronal temperatures similar to the $\mathrm{K}$ stars.

In order to probe the fainter Pleiades members as well, we 
have constructed composite X-ray spectra for different spectral types as a function of $v \sin i$. Model fits to the composite spectra confirm the results based on individual spectral fits. In addition, we have determined hardness ratios for nearly all the late-type stars. Plots of hardness ratio versus $L_{\mathrm{X}}$ and $L_{\mathrm{X}} / L_{\text {bol }}$ indicate generally higher ratios (i.e., harder spectra) with increased $X$-ray activity, at least among the $F$ and $G$ stars.

$X$-ray light curves have revealed a dozen strong flares in the PSPC data. Ten of these flares are associated with known Pleiades cluster members. During flares, $X$-ray luminosities rose by factors of 3-20 with rise times in the range 1-10 ks and decay times in the range 5-15 ks. The ratio of rise to decay time was typically $\sim \frac{1}{3}$, similar to long-duration flares observed by EXOSAT and Ginga (Pallavicini et al. 1990; Stern et al. 1992). Overall, the Pleiades flares are among the brightest $X$-ray flares ever reported on single, late-type main-sequence dwarfs.

In their analysis of a single PSPC field of the southwest portion of the Pleiades, Micela et al. (1995) detected three strong flares. This is consistent with our detection of 12 flares in three fields. By considering the number of detected flares, the number of observed stars, and the typical monitoring time of a single star, we estimate that strong, long-duration $\left(t \sim 10^{4} \mathrm{~s}\right)$ $\mathrm{X}$-ray flares occur approximately $1 \%$ of the time. This appears to be somewhat higher than the flaring frequency observed in the Hyades. In fact, Stern et al. (1994) detected only one flare in one PSPC field in the Hyades. This may be indicative of the overall decrease in activity and rotation rate from the age of the Pleiades to the age of the Hyades. On the other hand, Pleiades flares occur less often than on the UV Ceti-type flare stars (see Pallavicini et al. 1990).

In fact, Fleming et al. (1995) find that the flaring rate for dMe stars in the $\operatorname{ROSAT}$ all-sky survey may exceed $10 \%$. Thus, the low flaring rate in the Pleiades may appear somewhat surprising given the youth and high rotational velocities of the Pleiades stars. However, the flares we detect in the Pleiades are extremely energetic events, with most flare luminosities in excess of $3 \times 10^{30}$ ergs $^{-1}$. We detect very few less energetic flares. Hence, we may be substantially underestimating the flaring frequency.

The duration and peak luminosities of the Pleiades flares are reminiscent of strong flares detected on Algol by EXOSAT (van den Oord \& Mewe 1989) and Ginga (Stern et al. 1992) and on T Tauri stars in $\rho$ Oph (Montmerle et al. 1983) and Orion (Gagné et al. 1995). We have modeled one of the flares, associated with the late-K Pleiades star H II 1516, with a simple quasi-static cooling loop model. From the PSPC spectrum and light curve of the flare, we determine a peak emission measure in excess of $10^{54} \mathrm{~cm}^{-3}$ and infer a peak electron density $n_{e} Z$ $10^{11} \mathrm{~cm}^{-3}$. This suggests a large-scale flare event.

Since the Pleiades have been extensively monitored by both Einstein and ROSAT, we have been able to examine the variability of individual stars on timescales of $\sim 1$ yr between individual ROSAT exposures and over a decade between the Einstein and ROSAT missions. We find that on $\sim 1$ yr timescales, approximately one-fourth of the late-type Pleiades stars are variable by more than a factor of 2 . This is approximately the same fraction derived by us for the $T$ Tauri stars in the Orion Nebula region (Gagné \& Caillault 1994; Gagné et al. 1995). Based on Einstein observations of the $\rho$ Oph cloud, Montmerle et al. (1983) suggest that the flux variations in $T$ Tauri stars may be the result of continual flaring; however, the Pleiades ROSAT data are inconclusive.

On decade-long timescales, up to $40 \%$ of the late-type stars are variable by more than a factor of 2 . This result is in good agreement with the fraction determined by Schmitt et al. (1993) based on a similar analysis of the ROSAT all-sky survey data. Schmitt et al. (1993) have interpreted their findings as possible evidence of solar-like activity cycles in the Pleiades. However, we warn that the uncertainties involved in the determination of count-rate ratios are quite high because of the low detection threshold of the X-ray surveys. Moreover, we have compared data on only two timescales, and the level of variability is only marginally higher on the longer timescales. In order to determine the true nature of $X$-ray variability in late-type stars, frequent sampling over a much longer time line is required.

Note added in manuscript.- A strong flare detected in the PSPC data for the Hyades cluster member H II 2411 and reported by Preibisch \& Neuhäuser (1995) was not discussed herein because this star is not a Pleiades cluster member.

We wish to thank Jürgen Schmitt, Bob Stern, and Jeff Linsky for helpful discussions on X-ray variability and coronal heating. We would also like to thank the referee, Tom Fleming, for a thorough reading of this manuscript and for providing many constructive comments. This research was funded in part by NASA grants NAG5-1608 to the University of Georgia and NAGW-2968 and NAG5-1849 to the Harvard-Smithsonian Center for Astrophysics.
Avni, Y. 1976, ApJ, 210, 642

Baliunas, S. L., et al. 1995, ApJ, 438, 269

Bevington, P. R., \& Robinson, D. K. 1992, Data Reduction and Error Analysis

for the Physical Sciences (New York: McGraw-Hill), 104

Caillault, J. -P., \& Helfand, D. J. 1985, ApJ, 289, 279

Cully, S. L., Fisher, G. H., Abbott, M. J., \& Siegmund, O. H. W. 1994, ApJ, 435, 449

Dorren, J. D., \& Guinan, E. F. 1994, ApJ, 428, 805

Fisher, G. H., \& Hawley, S. L. 1990, ApJ, 357, 243

Fleming. T., Molendi, S., Maccacaro, T., \& Wolter, A. 1995, ApJS, in press

Gagné, M., Caillault, J.-P. 1994, ApJ, 437, 361

Gagné, M., Caillault, J.-P., \& Stauffer, J. R. 1995, ApJ, 445, 280

Haisch, B. M. 1983, in Astrophys. Space Sci. Libr. 102, Activity in Red Dwarfs

Stars, ed. P. B. Byrne \& M. Rodonò (Dordrecht: Reidel), 255

Jordan, C., \& Montesinos, B. 1991, MNRAS, 252, 21 P

Lampton, M., Margon, B., \& Bowyer, S. 1976, ApJ, 208, 177

Micela, G., Sciortino, S., Kashyap, V., Harnden, F. R., Jr., \& Rosner, R. 1995, ApJ, in press

Micela, G., Sciortino, S., Vaiana, G. S., Harnden, F. R., Jr., Rosner, R., \& Schmitt, J. H. M. M. 1990, ApJ, 348, 557

Montmerle, T., Koch-Miramond, L., Falgarone, E., \& Grindlay, J. E. 1983, ApJ, 269, 182

\section{REFERENCES}

Neuhäuser, R., Sterzik, M. F., Schmitt, J. H. M. M., Wichmann. R., \& Krautter, J. 1995a, A\&A, in press

Pallavicini, R., Tagliaferri, G., \& Stella, L. 1990, A\&A, 228, 403

Pfeffermann, E., et al. 1986, Proc. SPIE, 733, 519

Poletto, G., Pallavicini, R., \& Kopp, R. A. 1988, A\&A, 201, 93

Raymond, J., \& Smith, B. 1977, ApJS, 35, 419,(RS)

Rosner, R. Golub, L., \& Vaiana, G. S. 1985, ARA\&A, 23, 413

Schmitt, J. H. M. M. 1994, ApJS, 90, 735

Schmitt, J. H. M. M., Collura, A., Sciortino, S., Vaiana, G. S., Harnden, F. R., Jr., \& Rosner, R. 1990, ApJ, 365, 704

Schmitt, J. H. M. M., Kahabka, P., Stauffer, J. R., \& Piters, A. 1993, A\&A, 277, 114

Stauffer, J. R., Caillault, J.-P., Gagné, M., Prosser, C. F. \& Hartmann, L. W. 1994, ApJS, 91, 625 (Paper I)

Stern, R. A., Schmitt, J. H. M. M., Pye, J. P., Hodgkin, S. T., Stauffer, J. R., \& Simon, T. 1994, ApJ, 427, 808

Stern, R. A., Uchida, Y., Tsuneta, S., \& Nagase, F. 1992, ApJ, 391, 760

Trümper, J. 1992, QJRAS, 33, 165

van den Oord, G. H. J., \& Mewe, R. 1989, A\&A, 213, 245

Wilson, O. C. 1978, ApJ, 226, 379 